\title{
Review
}

Yun-Fei Li*, Zi-Long Kou, Jing Feng* and Hong-Bo Sun

\section{Plasmon-enhanced organic and perovskite solar cells with metal nanoparticles}

https://doi.org/10.1515/nanoph-2020-0099

Received February 9, 2020; revised March 16, 2020; accepted March 18, 2020

\begin{abstract}
Solution-processing thin-film solar techniques, such as organic solar cells (OSCs) and perovskite solar cells (PeSCs), hold great promise as cost-effective renewable energy sources with feasible large-scale manufacturing. However, these devices are suffering from the incomplete photon absorption and thereby cannot unlock the full potential of device efficiency despite their rapid development in recent decades. Incorporation of plasmonic metal nanoparticles (NPs) into the thin active layers has been considered as a breakthrough strategy to solve this inherent limit and represent an imperative milestone toward the highly efficient OSCs and PeSCs, arising from the significantly enhanced light absorption and electrical characteristics in fundamental. Herein, the recent advances in fabrication and incorporation strategies of plasmonic NPs are reviewed. The in-depth efficiency and stability enhancement mechanisms are investigated and highlighted. Meanwhile, potential strategies and perspectives for their further development of NP-based solutionprocessing OSCs and PeSCs are presented.
\end{abstract}

Keywords: organic solar cells; perovskite solar cells; plasmonics; metal nanoparticles.

\footnotetext{
*Corresponding authors: Yun-Fei Li, Tianjin Key Laboratory of Electronic Materials and Devices, School of Electronics and Information Engineering, Hebei University of Technology, 5340 Xiping Road, Beichen District, Tianjin 300401, People's Republic of China, e-mail: yfli@hebut.edu.cn; and Jing Feng, State Key Laboratory of Integrated Optoelectronics, College of Electronic Science and Engineering, Jilin University, 2699 Qianjin Street, Changchun 130012, People's Republic of China, e-mail: jingfeng@jlu.edu.cn

Zi-Long Kou: Tianjin Key Laboratory of Electronic Materials and Devices, School of Electronics and Information Engineering, Hebei University of Technology, 5340 Xiping Road, Beichen District, Tianjin 300401, People's Republic of China

Hong-Bo Sun: State Key Laboratory of Integrated Optoelectronics, College of Electronic Science and Engineering, Jilin University, 2699 Qianjin Street, Changchun 130012, People's Republic of China; and State Key Lab of Precision Measurement Technology and Instruments, Department of Precision Instrument, Tsinghua University, Haidian, Beijing, 100084, China. https://orcid.org/0000-0002-5676-403X
}

\section{Introduction}

The ongoing energy crisis combined with global warming and air pollution in recent decades induced with traditional fossil fuel has indicated the urgent requirement for the development of cost-effective, feasible, and largescale manufacture; environmental stability; and green energy technologies. To circumvent the energy crisis, renewable natural energy sources, such as solar energy, tidal power, and wind energy, have been explored as potential energy candidates [1]. The development of solar energy through photovoltaic energy conversion is the most promising alternative as sunlight is long-term sustainable, environmentally friendly, and abundant for energy production [2-7]. Inorganic solar cells, such as traditional silicon ( $\mathrm{Si}$ )-based or gallium arsenide-based, have been extensively reported to harvest sunlight and convert it into electrical power on a wafer-based scale, achieving high-power conversation efficiencies (PCEs) and superior environmental stability [2, 8]. Although inorganic solar cells possess the major share of current photovoltaic market nowadays, their expensive crystal growth techniques are not allowed to generate energy at a low price comparable to fossil fuels.

For the quest of cost-effective solar technologies, solution-processed techniques have been explored with shortened manufacturing time [9-11]. Thin-film solar cells, especially organic solar cells (OSCs) and perovskite solar cells (PeSCs), have appealed attention for years as the potential techniques to generate energy on a large scale with simple solution process [12-22]. In particular, they represent exceptional features such as compatibility with roll-to-roll process, flexibility, and lightweight, exceeding the inorganic solar cells [23-26]. Besides, the PCEs of OSCs and PeSCs have been rapidly developed in recent years [27-30]. To date, the PCEs of single-junction and tandemjunction OSCs have been significantly improved to $17.3 \%$ [31] and 16.35\% [32], respectively, whereas the PCE of PeSCs has reached to a relatively high value of $25.2 \%$ certified by the National Renewable Energy Laboratory (NREL) [https://www.nrel.gov/pv/cell-efficiency.html], equally matched to the Si-based solar cells. 
The dominant factor for realization of highly efficient solar cells is to balance the light absorption and the extraction of photogenerated excitons [33]. In general, the diffusion length of solution-processed organic semiconductors is much smaller than highly crystalline inorganic materials [34]. Therefore, the photoactive layers, namely, conjugated polymers or fullerene derivatives, in OSCs are commonly very thin with the order of $100 \mathrm{~nm}$ to trade off the low exciton collection rate induced by short diffusion length $[35,36]$. In this case, effective approaches should be explored to maximize the light harvesting across the solar spectrum range and achieve high PCEs with the reduced absorber thickness $[37,38]$. However, because of the small physical dimensions of OSCs, the prevailing strategies utilized in inorganic solar cells for light harvesting enhancement, such as textured pyramids, are not suitable in OSCs and need further modification. On the contrary, perovskite films possess long diffusion lengths up to $1 \mu \mathrm{m}$ and superior ambipolar transport ability [39], which relieves the stringent thickness rule. Although PeSCs have realized efficient light harvesting with planar device architectures as well as relatively thicker absorber $(\sim 500 \mathrm{~nm})$, yet in fact, the obtained PCEs of PeSCs are still below the theoretical limit, which requires further improvement [40].

Incorporation of plasmonic metal nanoparticles (NPs) is the most promising strategy to effectively improve the light harvesting in OSCs and PeSCs [41-47]. Metal NPs can substantially enhance the optical absorption of devices without influencing the device architecture due to the excitation of localized surface plasmon resonance (LSPR) and the far-field light scattering effect [48-50]. Besides, they have been also investigated to enhance the electrical characteristics, including exciton dissociation, charge transport, and collection in devices [51-54]. In this review, we address the characteristics of metal NPs and their applications in highly efficient OSCs and PeSCs. We discuss the plasmonic fundamentals of metal NPs along with the fabrication methods to control their sizes and shapes. We develop the evolution of device performance and the application of plasmonic NP-based OSCs and PeSCs and investigate the underlying enhancement mechanisms, as well as the future outlook in our perspective.

\section{Metal NP fundamentals}

Metal particles with nanometer dimensions have gained substantial attention in OSCs and PeSCs for decades due to their unusual optoelectronic and chemical properties
[55-57]. Given the diversified types of enhancements in plasmonic OSCs and PeSCs, it is necessary to comprehend the origins of these features. Fundamentally, when the size of metal NPs reduces to below the incident wavelength in the quasi-static limit, strong interactions can be perceived between the free electrons in the metal NPs and the electromagnetic radiation [58]. Plasmons are the collective oscillations of free electrons followed by the production of dipoles localized in the vicinity of the metal NP surfaces arising from the strong interactions with the incident lights [59]. Metal NPs have been employed in buffer layers, photoactive layers, or at the interface of OSCs and PeSCs to increase short-circuit current density $\left(J_{s c}\right)$ and PCE arising from the absorption enhancement [60-65]. Specifically, the incorporation of metal NPs can boost the light harvesting in OSCs and PeSCs by two mechanisms: a near-field plasmonic enhancement and an increase of the forward scattering cross section as shown in Figure 1 [3]. The plasmon resonance wavelength depends on the size, shape, distribution, and surrounding dielectric environment of metal NPs, determined by the fabrication methods [42, 66]. Plasmonic light trapping in OSCs and PeSCs and feasible strategies to control the size and shape of metal NPs are elaborated and summarized in this section.

\subsection{Physical properties}

Metal NPs show a strong ultraviolet (UV)-visible absorption, distributing within the absorption band of the active layer of OSCs and PeSCs, such as conjugated polymers and perovskite materials. Therefore, the employment of metal NPs either inside the buffer/active layers or between interfaces of OSCs and PeSCs devices can effectively enhance the light trapping by increasing the optical path length for light harvesting. The physical derivation of the enhanced absorption can ascribe to plasmonics, which are the coherent oscillations of the free conduction electrons excited by the incident light. Particularly, for metal NPs with dimensions similar or smaller than the wavelength of incident light, strong interactions are perceived between the free conduction electrons in the NPs and the electromagnetic electric fields. Coupled oscillations of electromagnetic light and the electrons oscillating are defined as LSPRs [67]. As the resonantly localized field amplification occurs, a dipolar field is generated at the vicinity of the plasmonic metallic NPs. This field results in strong near-fields outside the particles, increasing scattering cross section and electromagnetic light absorption by light trapping via plasmonics in both OSCs and PeSCs [4, 33]. 
A

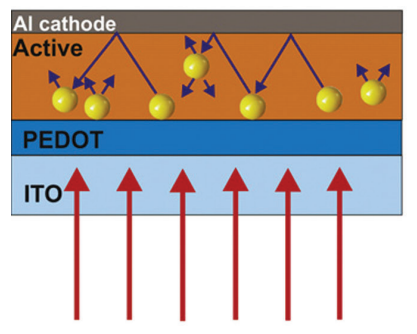

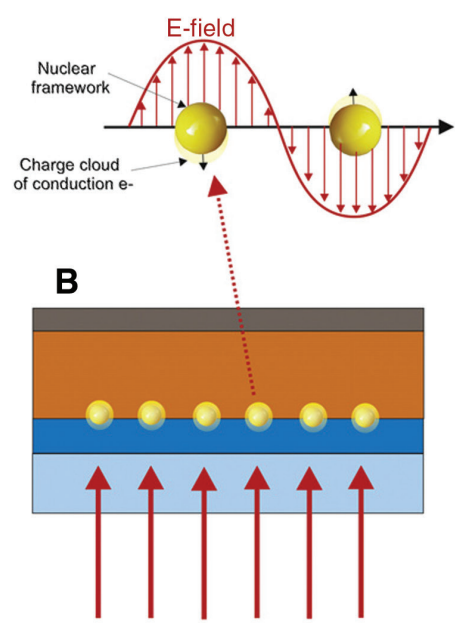

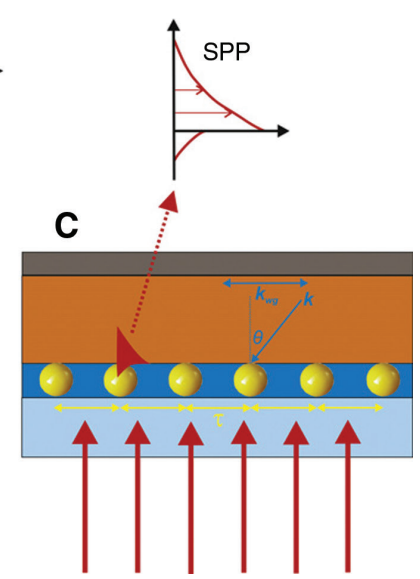

Figure 1: Plasmonic light trapping mechanism in metal NP-based OSCs.

(A) Plasmonic light scattering effect. (B) Localized surface plasmon resonance effect. (C) Surface plasmon polariton excitation of 2D periodic NP array at the NPs/photoactive layer interfaces. Reproduced with permission from Stratakis and Kymakis [3]. Copyright 2013, Elsevier.

If the size of the metal NP is below the incident wavelength, the scattering $\left(\sigma_{\mathrm{sc}}\right)$ and absorption $\left(\sigma_{\text {abs }}\right)$ cross sections are given from the quasi-static approximation via the following equations [68]:

$$
\begin{gathered}
\sigma_{\text {sc }}=\frac{1}{6 \pi}\left(\frac{2 \pi}{\lambda}\right)^{4}|\alpha|^{2} \\
\sigma_{\text {abs }}=\frac{2 \pi}{\lambda} \operatorname{Im}(\alpha)
\end{gathered}
$$

where $\alpha=3 V\left(\varepsilon_{p}-\varepsilon_{m}\right) /\left(\varepsilon_{p}+2 \varepsilon_{m}\right)$ is the polarizability of the metal NP, and $\lambda$ is the wavelength of incident light. For spherical NPs, the polarizability can be calculated via $\alpha=4 \pi \mathrm{r}^{3}\left(\varepsilon_{p}-\varepsilon_{m}\right) /\left(\varepsilon_{p}+2 \varepsilon_{m}\right)$. Here $\varepsilon_{p}$ and $\varepsilon_{m}$ are the permittivity of the plasmonic NP and the embedding semiconductor medium, and $V$ is the volume of the metal NP, respectively. Figure 2 demonstrates the electromagnetic field distribution of plasmonic metal NPs influenced by the size and surrounding medium in detail [69]. It is well-defined that $\sigma_{\text {sc }}$ relies on both the size of the metal NP and the surrounding local dielectric semiconductor. It can be concluded that when the light frequency is such that $\varepsilon_{p} \approx-2 \varepsilon_{m}$, the metal NPs exhibit maximum polarizability and LSPR, which is nonpropagating excitations of free electrons within metal NPs. One exceptional characteristic of LSPRs is the enhanced electromagnetic field due to the strong local resonance, resulting in efficient light concentration with enhancement factors as high as 100. Plasmonic fields surrounding the metal NPs damp exponentially related to the distance from the NPs. The frequency of the resonance and decay lengths of the electrical field are determined by the metal NP materials, the dimensions, geometric shapes and configurations of the metal NPs, and the optical parameters of the surrounding environment, which renders process tunability.

On the basis of (1) and (2), the particle dimension certainly takes charge of the extinction process, determining whether metal NPs function as scattering centers or local electromagnetic field enhancers or both. Figure 1 classifies the physical principles resulting in the enhancements in plasmonic OSCs. The enhancements of device performance can be ascribed to the modifications in optoelectrical properties. Specifically, smaller metal NPs with diameter of 5-20 $\mathrm{nm}$ can be utilized as subwavelength antennas. In this case, enhanced absorption dominates owing to LSPR excitation. The local plasmonic field couples to the photoactive layer in OSCs and PeSCs, which enhances absorption and accordingly exciton dissociation. In contrast, relatively larger diameter particles $(>50 \mathrm{~nm})$ perform as subwavelength scattering in photovoltaic applications that couple and trap freely propagating incident photons into active layer.

Under this circumstance, enhanced absorption is dominated by the increased optical path length, induced by the re-emitted light within the device. However, if the particle is too large, multipole oscillations will be strengthened, which reduces the scattering efficiency of $Q_{\mathrm{sc}}$. Besides, when particle dimension exceeds the Rayleigh approximation, the resonance will broaden and redshift. Overall, smaller particles mainly absorb the photons, whereas larger ones scatter light more efficiently. The scattering efficiency $\left(Q_{\mathrm{sc}}\right)$ can be described by the relation: $Q_{\mathrm{sc}}=\sigma_{\mathrm{sc}} /\left(\sigma_{\mathrm{sc}}+\sigma_{\mathrm{abs}}\right)$ [40]. While plasmonic 

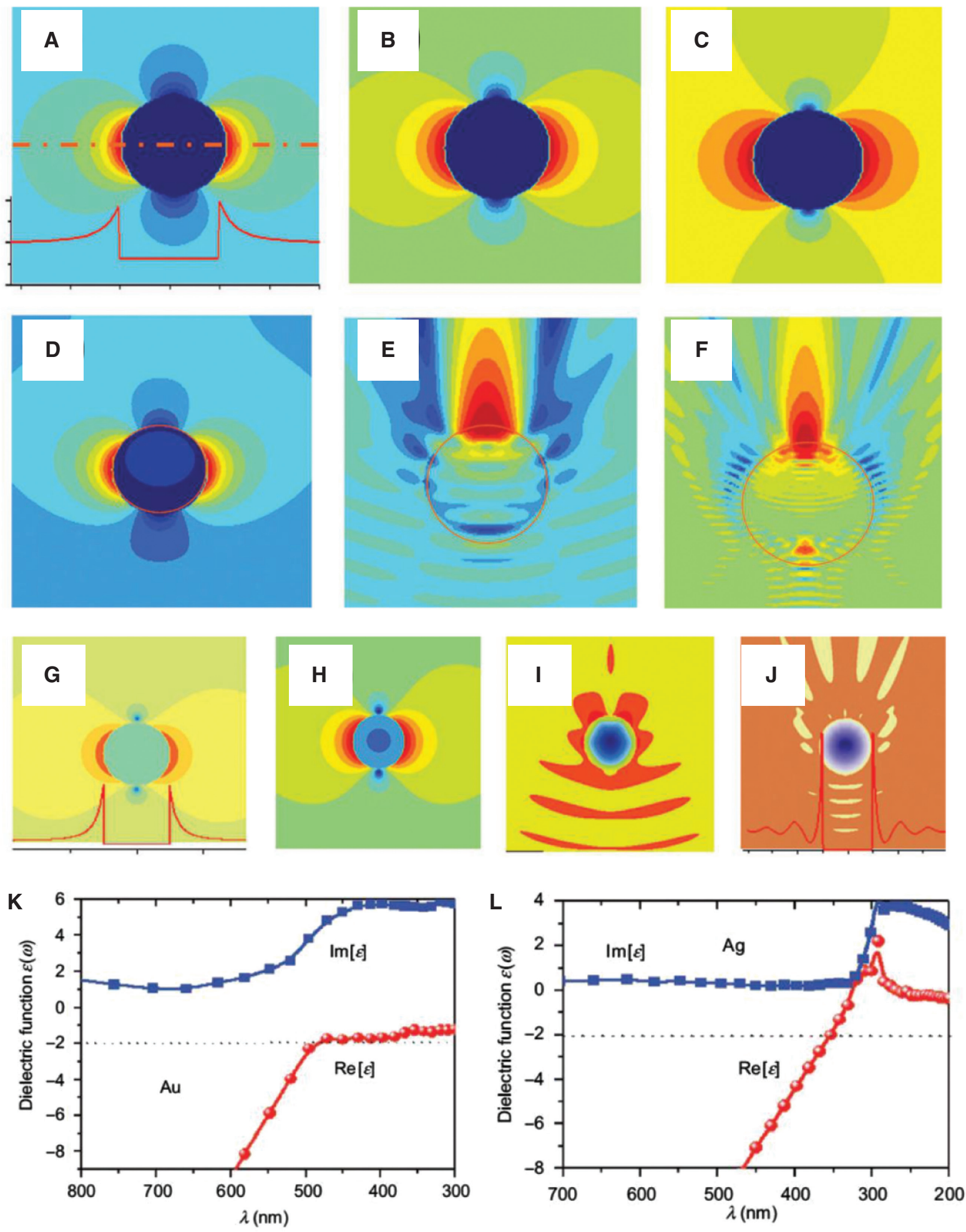

Figure 2: Electric field distribution and dielectric function for metal NPs.

Electric field distribution of plasmonic metal NPs influenced by the size (A-F) and surrounding medium (G-J), respectively. (K-L) Dielectric function for small Au and Ag NPs. Reproduced with permission from Fan et al. [69]. Copyright 2014, Nature Publishing Group.

NPs provide efficient near-field enhancement and scattering, it is necessary to guarantee that the absorption by the plasmonic NPs is minimal, especially for smaller particles.
Absorption by the plasmonic NP cannot produce photocurrent; thus, $\sigma_{\mathrm{sc}}$ should outweigh $\sigma_{\mathrm{abs}}$ for valid utilization of the plasmonic NPs. 
At the distance of $r$ from the center of spherical metal NPs, the maximum field enhancement from LSPR embedding in a nonabsorbing semiconductor can be expressed via the relation [70]:

$$
\eta_{\max }=\left|1+\frac{\alpha(\omega)}{2 \pi r^{3}}\right|^{2}
$$

where $\omega$ is the frequency of the incident light. The field enhancement $\left(\eta_{\max }\right)$ is essentially an upper limit approximation on account of the nonabsorbing environment.

Furthermore, metal NPs can also consist of periodical array applied in organic photovoltaics (OPVs) as shown in Figure 1C. Here, incident electromagnetic wave excites resonant scattering modes, which is described as surface plasmon polaritons (SPPs) at the interface of NP-photoactive layer. Besides, two-dimensional (2D) metal NPs array can supply in-plane momentum for scattered light to be coupled to propagation or waveguide modes [71, 72]. The in-plane momentum can be calculated by the equation of $q \frac{2 \pi}{\tau}$, where $\tau$ is the pitch and $q(= \pm 1, \pm 2, \pm 3, \ldots)$ is the diffraction order. In this case, incoming light can couple into waveguide modes through momentum matching under the following conditions [73]:

$$
k_{\mathrm{wg}}=k \sin \vartheta+q \frac{2 \pi}{\tau}
$$

where $k_{\text {wg }}$ represents the momentum for coupling; $k \sin \vartheta$ is the in-plane momentum of the incident light. The geometrical morphology of the metal NPs arrays, such as their pitch and size, should be well-designed and controlled to fulfill the optimal momentum conversion and further absorption enhancement in devices.

Indeed, Nishijima et al. [74] have investigated the effect of random and periodic particle configurations on the light field enhancement of plasmonic resonance. An enhancement of extinction and a broader plasmon resonance were observed in NPs with increased disorder, which could be ascribed to grating-like losses arising from diffraction. Finite difference time domain (FDTD) modeling calculations demonstrated an augmentation of the enhancement by more than two orders of magnitude for the random nanodisk patterns; however, those hotspots were more sparsely distributed. The randomly distributed NPs are one of the cheapest approaches to enhance light harvesting of solution-processed solar cells, favored for industrial applications.

Notably, the electromagnetic resonance spectrum of noble metal NP is distributed ranging from visible to infrared (IR) region, which is attractive to OSCs and PeSCs.
Therefore, silver (Ag) and gold $(\mathrm{Au})$ NPs have been hotly pursued in devices for decades. Indeed, other metal NPs can show plasmonic properties as well; specifically, aluminum (Al) NPs exhibit limited metal absorption due to their effectively reduced reflection losses from the high resonant frequency $[33,75]$. Besides, copper $(\mathrm{Cu}) \mathrm{NPs}$ with appropriate capping agents to improve stability in air have also been employed in OSCs [76, 77].

According to the location of plasmonic metal NPs employed in the front or rear side of the device architecture, their integration in OSCs and PeSCs can be divided into the following categories: charge transport layers, photoactive, and between interfaces [33, 71]. These categories are essentially dissimilar in principle concerned with the plasmonic NP-based absorption enhancement. Positioning the metal NPs in the front or rear side of the devices is a potential choice for plasmonic scattering as they can effectively isolate the electric activity of the active layer and the optical properties of the NPs and avoid destructive interference. Metal NPs can be integrated into the charge transport layers, photoactive layer, or between interfaces in OSCs. It is also noteworthy that the incorporation of NPs into the photoactive layer can result in the improvement of structural durability with slower degradation rate under the illumination radiation [78], which causes a further performance enhancement. The enhancements of device performance and changes to device physical mechanisms in plasmonic OSCs were investigated to derive from the modifications in morphological, electrical, and optical characteristics [79]. Specifically, modifications in electrical properties of OSCs, such as exciton generation, hot carrier transfer, and charge extraction, played an important role in performance enhancement induced by improved opencircuit voltage $\left(V_{\text {oc }}\right)$, fill factor $(\mathrm{FF})$, and photocurrent generation. For PeSCs, only few research efforts have been devoted into plasmonics applications so far, which are generally similar to those explored in OSCs. It is expected that PeSCs with plasmonic NPs will be addressed as the thorough comprehension of the electrical and optical effects. Besides, polymer solar cells (PSCs) should receive particular concern because of their environmental instability, especially for the incorporation of $\mathrm{Al}$ or Ag NPs [80]. The effects of plasmonic metal NPs in both OSCs and PeSCs and the resultant enhancements will be discussed in more detail and shown in the following sections.

\subsection{Fabrication methods of metal NPs}

Structural parameters of metal NPs make a great difference to manipulate the excitation, trap, and coupling process 
of photons in OSCs and PeSCs. To date, considerable numbers of fabrication technologies have been exploited for the plasmonic NPs with controllable morphology [46, 81, 82]. The NPs with versatile shapes and morphologies show distinct optical responses arising from the collective oscillations giving rise to LSPR and plasmonic scattering. The availability of microfabrication and nanofabrication methods with large scale and low cost is a crucial point to the commercial application of photovoltaic module. In this section, we will discuss several fabrication methods to form versatile and scalable metal NPs with a wide range of sizes, shapes, and high yields applied in OSCs and PeSCs, such as chemical synthesis technologies, laser ablation methods, and thermal annealing routes.

Regarding the chemical synthesis technologies, the metal NPs are synthesized mainly by the reduction of precursors with controlled aspect ratio. Brust et al. [83] and Turkevitch [84] aqueous preparation methods are commonly utilized for the fabrication of NPs smaller than $10 \mathrm{~nm}$ and $10-40 \mathrm{~nm}$ in diameter up to a limit, respectively. Ligand exchange step is always followed in this method, on account of avoiding aggregation in the growth process [85]. For example, Au halides are generally used as precursors for the synthesis of Au NPs, which are obtained by dissolving bulk $\mathrm{Au}$ in aqua regia to form chloroauric acid or in metal cyanide to form Au chloride. In this case, the introduction of an appropriate reduction agent is significant to prepare uniformly shaped NPs with narrow size distribution $[46,86]$. Specifically, citrate is often used to function as the reduction agent in fabrication of $\mathrm{Au}$ NPs with spherical morphology $[87,88]$. By adjusting the concentration ratio of citrate and $\mathrm{Au}$ precursor, the size of $\mathrm{Au}$ sphere can be regulated, which is indicated by the color variation of the solutions as shown in Figure 3A. The light scattering effects of incorporated NPs into the poly(3,4-ethylenedioxythiophene):poly(styrenesulfonate) (PEDOT:PSS) layers are presented in Figure 3B.

Besides, laser ablation methods are usually employed to prepare metal NP patterns in OSCs and PeSCs in order to excite LSPR or plasmon scattering [89]. In this method, the solid target dispersed in a liquid medium is treated with pulsed picosecond and femtosecond laser [90] ablation to obtain the plasmonic NPs, which are appealing candidates for incorporation in OSCs and PeSCs, especially for the photoactive layer [91, 92]. This method features the advantage of easily producing plenty of NPs free of unnecessary reagents such as stabilizers, surfactants, and passivation layers, when compared to the chemical synthesis methods $[93,94]$. It is noteworthy that surfactants on the surface of NPs possibly accelerate unwanted exciton quenching through nonradiative energy transfer between the NPs and active layers; thereby decreased plasmonic effect is observed. Photoconversion efficiency has been reported to be decreased after the addition of chemically synthesized $\mathrm{Au}$ NPs without capping in the photoactive layer [94]. In contrast, laser-ablated Ag NPs doped in the active layer of OSCs can cause enhanced photodegradation stability [54]. However, it is hard to obtain monodispersed colloids of metal NPs with this technique [90, 91]. Stratakis and Kymakis reported Au NPs formed by femtosecond laser ablation with initial colloidal solution of Au metallic target dispersed into a Pyrex cell and covered absolute ethanol [92]. This technique supplies massive NPs with neither counter-ions nor surface-active substances.
A

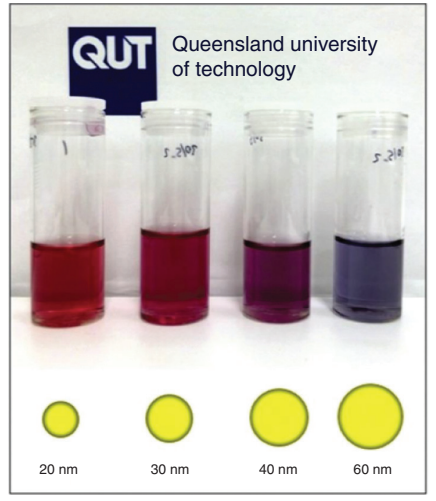

B

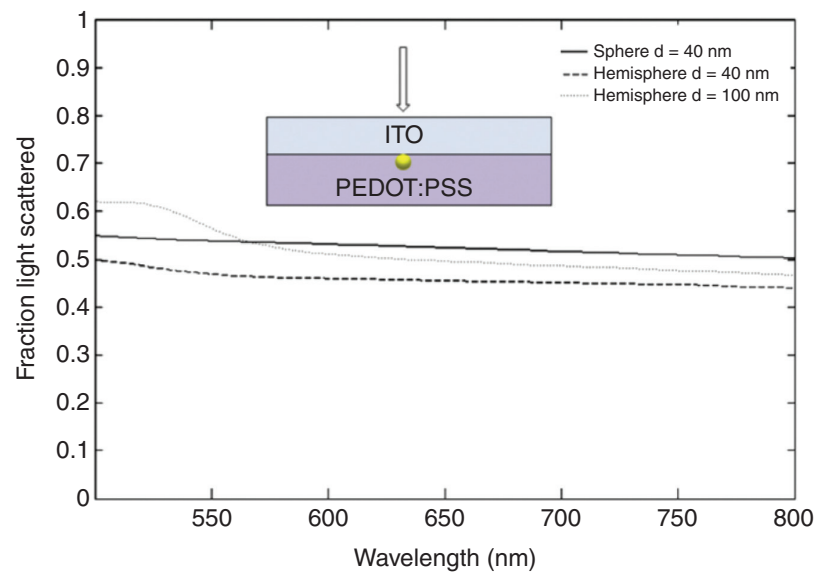

Figure 3: Characteristics of Au NPs synthesized with the chemical technology.

(A) Gold NPs solutions with sizes of 20,30, 40, and $60 \mathrm{~nm}$. (B) The light scattering effects of incorporated Au sphere with diameter of $40 \mathrm{~nm}, \mathrm{Au}$ hemisphere with diameter of $40 \mathrm{~nm}$, and Au hemisphere with diameter of 100 nm into the PEDOT:PSS layers. Reproduced with permission from Notarianni et al. [46]. Copyright 2014, Elsevier. 
Kubiliu-te et al. demonstrated ultrapure, water-dispersed $\mathrm{Au}$ NPs produced by fs-white light continuum generation in colloidal solutions to precisely control the size [95]. The narrow-size distributed NPs with preferentially spherical shape showed enhanced stability without sedimentation for months.

Another captivating method for the production of metal NPs is thermal annealing route combined with traditional deposition techniques to fabricate various NPs patterns on different positions in OSCs and PeSCs [96-98]. Mallik et al. [99] reported bimetallic Au and Ag NPs with a unique core-shell structure with UV-photoactivation technique. In this method, Au NPs were functioned as seed particles and catalyzed the reduction of Ag ion under UV irradiation to produce the bimetallic NPs with $\mathrm{Au}_{\text {core }}-\mathrm{Ag}_{\text {shell }}$ configuration [99]. Xu et al. [100] demonstrated a simple coevaporation of $\mathrm{Au}$ and $\mathrm{Ag}$ thin films onto the indium tin oxide (ITO) substrate, followed by the vacuum thermal annealing method to create $\mathrm{Au}-\mathrm{Ag}$ alloy NPs with tunable molar ratio.

\section{Plasmonics in 0SCs}

Metal NPs incorporated with the single or tandem OPV cells substantially improve the optical absorption of devices via the light trapping induced predominantly by the excitation of near-field LSPR with the small NPs or the far-field light scattering with the large NPs [48]. Besides, the application of metal NPs in OSCs has been demonstrated to improve the electrical characteristics, including exciton dissociation, charge transport, and collection [51-54]. These effects enhance the device performance in various configurations of the plasmonic NPs, when employed in the photoactive layer or the buffer layer (charge transport layer), between the interface as shown in Figure 4 [101]. Generally, the optimization of device performance is achieved through adjusting the materials, geometric shapes, size distributions, and topological distributions of plasmonic NPs in the device architecture, which can be guided by the theoretical simulations, such as FDTD modeling $[5,60,102]$. Here, we demonstrate the light absorption enhancements with plasmonic NPs in OSCs in detail.

\subsection{Optical absorption}

When incorporated plasmonic NPs with size much smaller than the wavelength of incident light, the enhanced absorption in the OSCs originates predominantly from the excitation of near-field LSPR mode. Plasmonic metal NPs are investigated to be effective on improving the device performance of OSCs when doped into multiple functional layers within the device architecture.

Theoretically, incorporation of the plasmonic metal NPs into photoactive layers is the optimum strategy, as it can maximize the contribution of LSPR to the light harvesting under this circumstance. Deliberated design is needed in positioning the NPs in photoactive layer as exciton quenching, nonradiative decay, and charge carrier recombination can occur $[51,54,103,104]$ on the surface of unpassivated NPs. Packing NPs inside an inert cladding layer with core-shell structure to passivate the chemically synthesized NPs as well as laser-ablated NPs can effectively alleviate this issue $[105,106]$. Spyropoulos et al. [92] presented a 40\% PCE enhancement in OSCs after embedding laser-ablated Au NPs into the photoactive of poly(3-hexylthiophene):phenyl-C61-butyric acid methyl ester (P3HT:PCBM) in 2012. Notably, the spectral ranges of incident photon to charge carrier efficiency (IPCE) enhancement and quantum efficiency were investigated to match with the LSPR region of Au NPs doped in the photoactive medium. Therefore, the efficiency enhancement can be ascribed to improved light harvesting and subsequent exciton generation arising from LSPR and scattering effects. Xie et al. [107] demonstrated highefficiency PSCs with an efficiency enhancement of $22 \%$ via incorporating poly(ethylene glycol) (PEG)-capped $\mathrm{Au}$ NPs of sizes 18 and $35 \mathrm{~nm}$ in both anodic buffer layer and photoactive layer simultaneously in 2011 shown in Figure 5A. The incorporation of PEG-capped Au NPs into anodic buffer layer of PEDOT:PSS led to enhanced hole collection, whereas their incorporation into photoactive layer of P3HT:PCBM resulted in the optical absorption enhancement and charge transport balance. Specifically, the absorption enhancement within the photoactive layer can be assigned to the strong near-field LSPR effect penetrated in the photoactive polymers. Meanwhile, coupling between plasmonic NPs in individual buffer and photoactive layers was not detected. These findings indicated that incorporation plasmonic NPs in different configurations simultaneously can be utilized to fulfill larger enhancement of device performance, which provided guidance for designing high-efficiency OSCs with NP incorporation.

Integration of NPs into the buffer layer (charge transport layer) is one of the easy, effective, and nondestructive approaches to harness the plasmonic effects. Incident light at wide angles can be scattered into the photoactive layer and fulfill the light trapping, thereby increasing the light path length within the OSCs and possibly resulting in total internal reflection. Besides, the scattered light can 


\section{A}

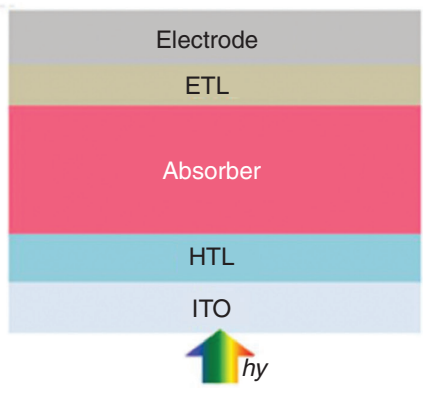

B

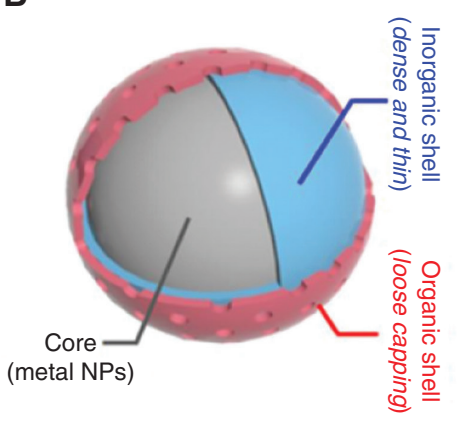

E

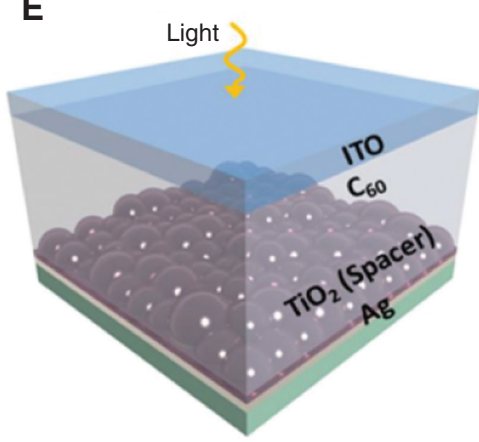

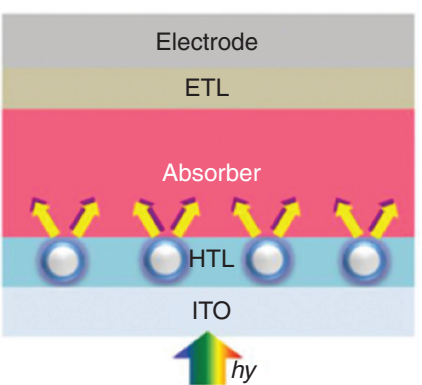

C

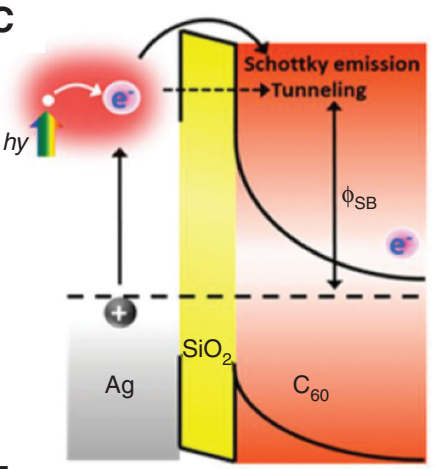

$\mathbf{F}$

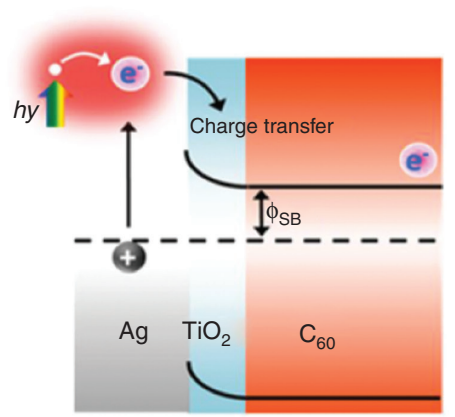

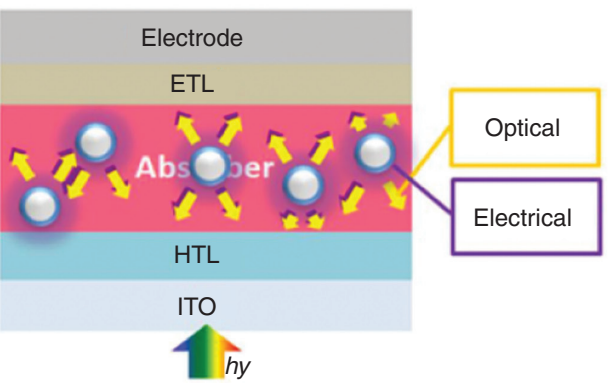

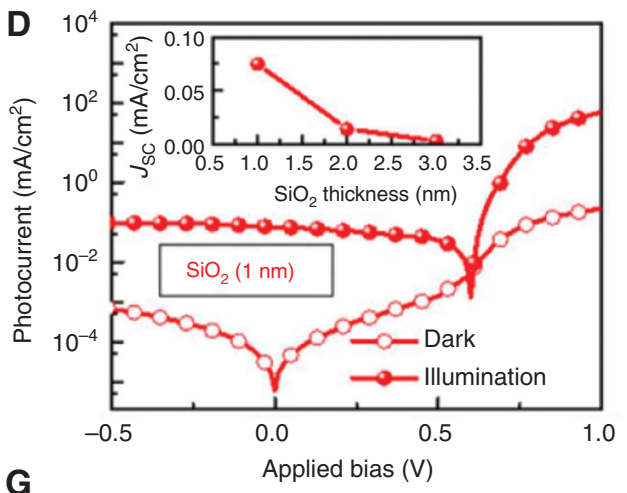

G

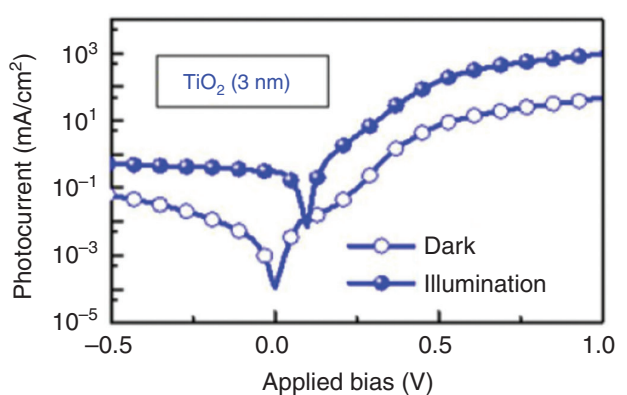

Figure 4: Multiple synergistic effect of optical absorption enhancement and electrical characteristics occurred in OSCS with the incorporation of plasmonic metal NPs.

(A) Schematic illustration of the plasmonic solar cell architectures with different spatial locations of metal nanostructures. (B) Core-bishell design concept for metal nanostructures embedded within an active layer. (C) Nanoplasmonic device structure consisting of $\mathrm{Ag}_{\mathrm{T}} / \mathrm{TiO} / \mathrm{C}_{60}$ and $\mathrm{Ag} / \mathrm{SiO}_{2} / \mathrm{C}_{60}$ stacked films to simulate the core-bishell designs of Ag@TiO $@ \mathrm{~Pa}$ and $\mathrm{Ag} @ \mathrm{SiO}_{2} @ \mathrm{~Pa}$, respectively. (D) Energy diagram of electron excitation based on between-band transfer for the $\mathrm{SiO}_{2}$ interface. (E) Energy band diagram of metal-insulator-semiconductor for the device with $\mathrm{TiO}_{2}$ layer. Current-voltage characteristics in the dark and under simulated AM1.5G solar illumination of a typical device with $1 \mathrm{~nm}$ of $\mathrm{SiO}_{2}(\mathrm{~F})$ and $3 \mathrm{~nm}$ of $\mathrm{TiO}_{2}(\mathrm{G})$ between nanostructured $\mathrm{Ag}$ film and $\mathrm{C}_{60}$. The inset of $(\mathrm{F})$ demonstrated the dependence of $\mathrm{SiO}_{2}$ thickness on short-circuit current. Reproduced with permission from Yao et al. [101]. Copyright 2019, American Chemical Society.

potentially excite SPPs along the intervening planar surfaces. Incorporation of plasmonic metal NPs in multiple layers can be an effective strategy to further improve the device performance.

Wu et al. [108] incorporated Au NPs into the PEDOT:PSS buffer layer and systematically investigated the influence of plasmonic structure on the performance of OSCs shown in Figure 5B. The enhancement degree of light absorption in the plasmonic-enhanced OSCs mainly depended on the local enhancement of LSPR-induced local field at the vicinity of Au NPs. The rate of exciton generation was improved; meanwhile, the lifetime of photogenerated excitons in the active layer was reduced by LSPR effect.
Accordingly, interactions between the plasmons and the photogenerated excitons led to an increased probability of exciton dissociation, decreasing the recombination level of geminate excitons. Yao et al. added nanoprisms both in the front and rear side, specifically PEDOT:PSS layer and $\mathrm{C}_{60}$-bis layer, and permitted an enhancement of $18 \%$, from $7.7 \%$ to $9.0 \%$ as shown in Figure 6 [110].

Embedding NPs at interfaces within devices can excite LSPRs at their surrounding along with SPPs along the planar surfaces. Thus, the embedded NPs can simultaneously enhance absorption and collect charge carriers in OSCs. Stenzel et al. presented the ITO-Cu phthalocyanine-indium sandwich structures combined with metal 

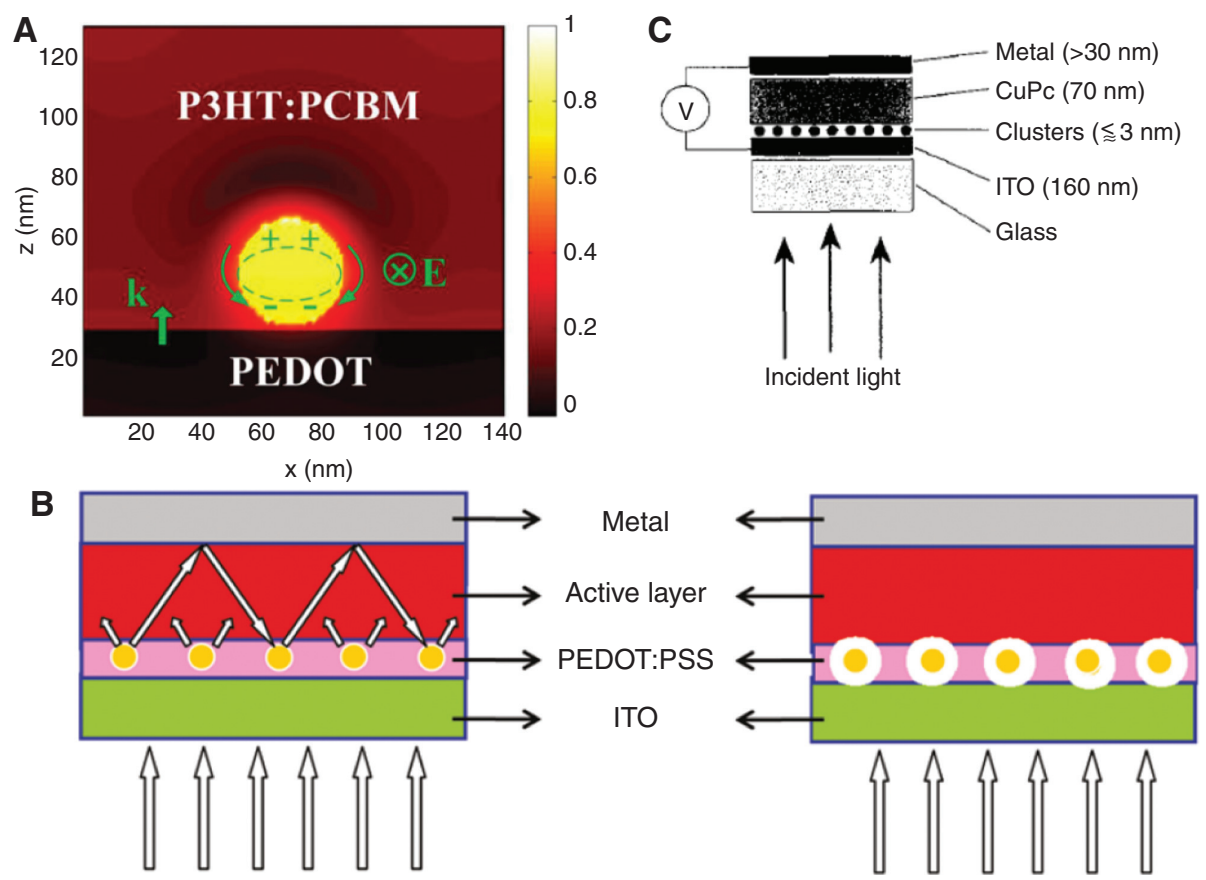

Figure 5: Incorporation of plasmonic metal NPs into different positions in OSCs.

(A) Photoactive layer. (B) Buffer layer. (C) Between interfaces. Reproduced with permission from Xie et al. [107], Copyright 2011, American Institute of Physics; Wu et al. [108], Copyright 2011, American Chemical Society; Stenzel et al. [109], Copyright 1995, Elsevier.

nanoclusters shown in Figure 5C [109]. The integral photocurrent enhancement by a factor of nearly three was first recorded by blending $\mathrm{Cu}$ clusters, which was assigned to the LSPR and interband transitions in the $\mathrm{Cu}$ clusters. A breakthrough occurred in a study by Rand et al. [111] in 2004 when an array of 5-nm-diameter NPs was employed into the intermediate layer in tandem thin-film OSCs. A significant efficiency enhancement by a factor of more than 2 was demonstrated, which was assigned to the enhanced optical field induced by both LSPR and light scattering. Furthermore, this enhanced field existed far from the particle surface, reaching a long range up to $10 \mathrm{~nm}$ from the center of the NPs. Later on, Xu et al. [100] employed thermally annealed $\mathrm{Au}-\mathrm{Ag}$ alloy NPs in OSCs with adequate molar ratio and obtained a 19\% enhancement with PCEs. The enhancement can be ascribed to the amplified electric field at the NP surface arising from the near-field LSPR coupling.

In OSCs, incident light penetrates the substrates, electrodes, and buffer layers, which are finally absorbed by the thin photoactive layer. Enhanced incident photon absorption can be realized by employment of arrayed or random metal NP scatters in devices along the incident direction. For larger-diameter NPs employed in OSCs, far-field scattering effects play the main role in coupling incident light into photoactive layer and enhancing absorption in devices. Because of the light scattering, the plasmonic NPs with large scattering cross section can supply more effective path lengths, trap light, and improve in-coupling efficiency in devices as shown in Figure 7 [112-118]. The scattering effect can be adjusted by optimizing the geometric parameters, including the size, shape, distribution, and surrounding medium of NPs in OSCs.

In a related article, Wang et al. proposed enhanced performance of OSCs with employment of large truncated octahedral Au NPs with an approximate size of $70 \mathrm{~nm}$ into a photoactive layer of poly(3-hexylthiophene):[6, 6]-phenyl C70 butyric acid methyl-ester (P3HT:PC ${ }_{70} \mathrm{BM}$ ), and poly[N9'-hepta-decanyl-2,7-carbazole-alt-5,5- $\quad\left(4^{\prime}, 7^{\prime}\right.$-di-2thienyl$2^{\prime}, 1^{\prime}, 3^{\prime}$-benzothiadiazole) (PCDTBT:PC $\left.C_{70} \mathrm{BM}\right)$, and poly $\{[4$, 4'-bis(2-ethylhexyl) dithieno(3,2-b:2',3'-d)silole]-2,6diylalt-[4,7-bis(2-thienyl)- 2,1,3-benzothiadiazole]-5,5'-diyl\} (SiPCPDTBT:PC $\left.{ }_{70} \mathrm{BM}\right)$ in 2011 [61]. In this case, the PCE enhanced from $3.54 \%$ to $4.36 \%$ for P3HT:PC ${ }_{70} \mathrm{BM}$-based devices, from $5.77 \%$ to $6.45 \%$ for PCDTBT:PC ${ }_{70} \mathrm{BM}$-based devices, and from $3.92 \%$ to $4.54 \%$ for Si-PCPDTBT:PC70BMbased devices with $5 \mathrm{wt} \% \mathrm{Au}$ NPs doping ratio, which can be assigned to the enhanced light absorption on account of scattering effects from LSPR modes. Wang et al. performed a comparative study of different NP shape incorporated in poly[N-999-hepta-decanyl-2,7-carbazole-alt-5,5-(49,79di-2-thienyl- 29,19,39-benzothiadiazole)]:[6,6]-phenyl C71 butyric acid methyl-ester (PCDTBT:PC ${ }_{71} \mathrm{BM}$ ) devices, specifically for the shape-controlled Ag nanoplates and Ag NPs with well-defined size [119]. Power conversation efficiency enhancement of $37.5 \%$ and $25 \%$ has been demonstrated for Ag NPs and Ag nanoplates-based devices, respectively. 


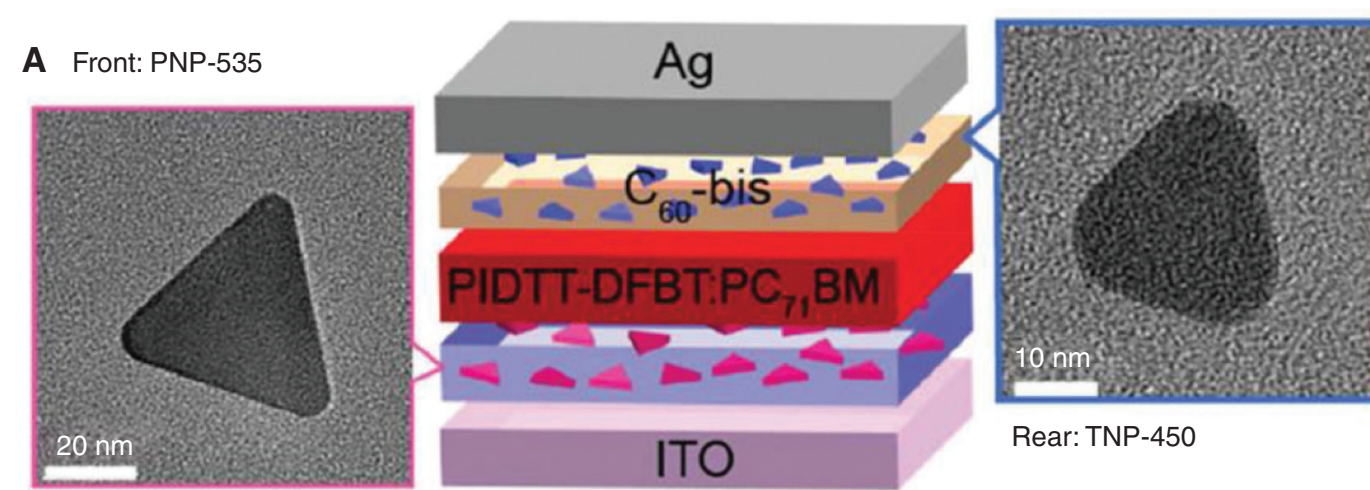

B

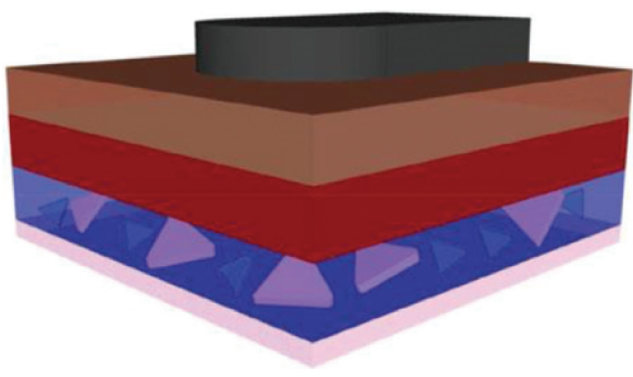

D

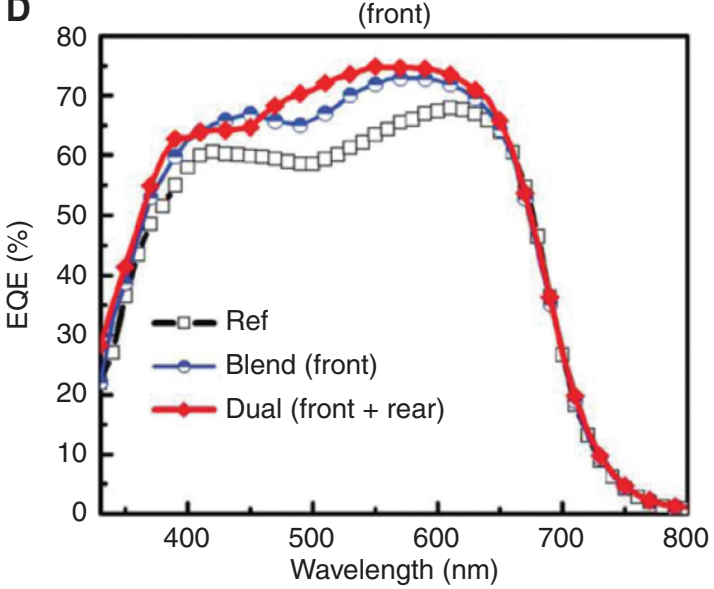

C

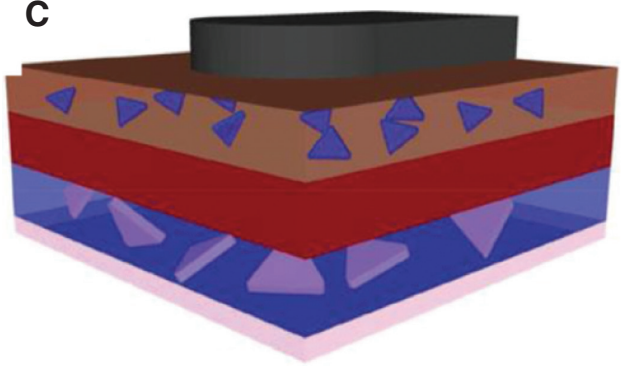

E

Dual

(front + rear)

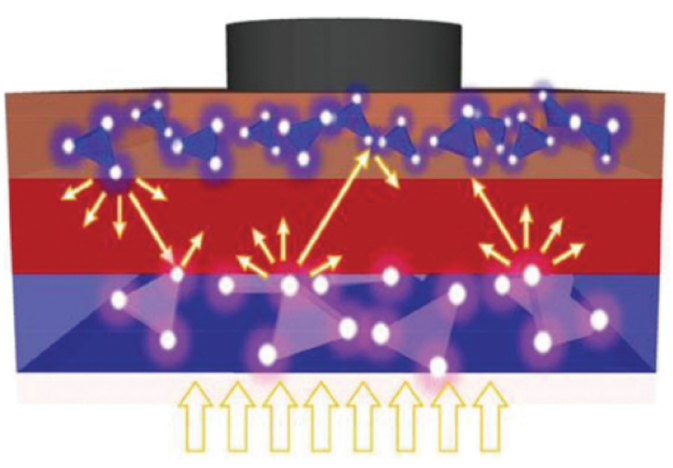

Figure 6: Schematic of materials and nanoprisms used in the dual plasmonic BHJ device and corresponding device performance. (A) Schematic diagram of the device configuration with dual incorporation of metal NPs into PEDOT:PSS and $\mathrm{C}_{60}$-bis layer, respectively. (B-E) Device performance comparison with combination metal NPs in different layers. Reproduced with permission from Yao et al. [110]. Copyright 2014, Wiley-VCH.

The advantages of the nanoplates can be ascribed to their shape, which benefited from the efficient scattering and light trapping in photoactive layers. Later on, Kalfagiannis et al. [120] investigated the effect of NP positions deposited between anode and buffer layer or between photoactive layer and cathode. Placing NPs on top of ITO anode led to an efficiency enhancement of $17 \%$, connected to the light scattering and LSPR effects, while locating NPs between P3HT:PCBM and Al cathode exhibited 25\% higher photocurrent generation through the reduction of buffer layer resistance. In particular, this study also presented that scattering effect is not significantly influenced by the angle of incidence or the polarization of light. The improved electromagnetic fields induced by LSPR were considered to benefit to enhance the rate of exciton generation and probability of exciton dissociation [120]. Baek et al. proposed a novel metal-metal core-shell nanocube (NC) structure for efficient plasmon-assisted PTB7:PC ${ }_{70} \mathrm{BM}$-based OSCs with a PCE of 9.2\% [121]. The Au-Ag core-shell structure demonstrated a high scattering efficiency at long wavelength and minimized blue shift, when compared to uncovered Au NPs (Figure 8).

In addition to the LSPR and scattering effects induced by metal NPs in OSCs, the excitation of SPP modes with 


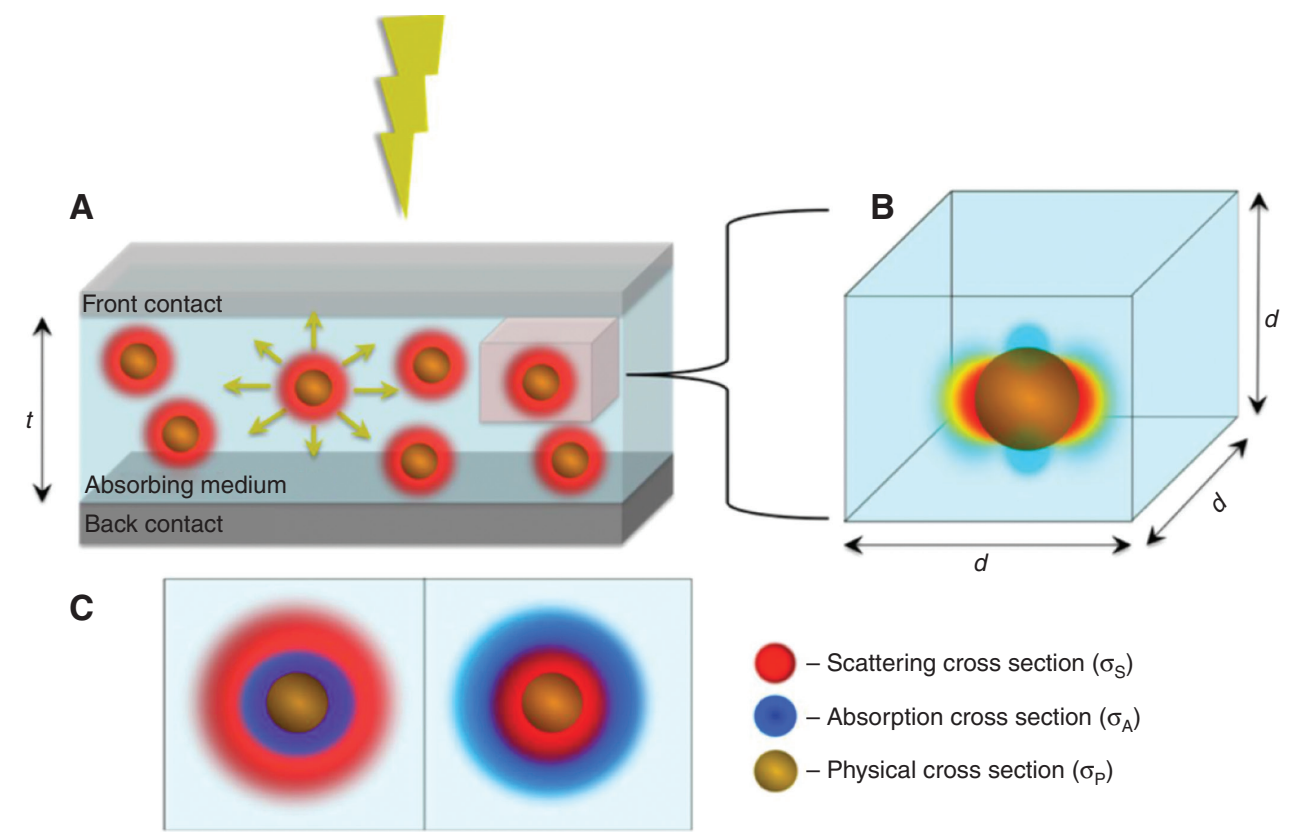

Figure 7: Diagram of cross-section light scattering effect of the metal NPs.

(A, B) Schematic diagram of randomly distributed NPs dispersed in the absorbing medium. (C) Cross section of the unit cell where the scattering cross section exceeds the absorption cross section (left), and absorption cross section surpasses the scattering cross section (Right) of the NPs. Reproduced with permission from ref. [112]. Copyright 2016, American Chemical Society.

incorporation of metallic gratings also plays crucial roles in effective light trapping. Surface plasmon polariton is the guided electromagnetic wave traveling along the metal/ dielectric interface. Through surmounting the mismatch between the momentum of in-plane SPP and incident photos with incorporation of metallic grating electrodes, the SPP modes can be excited, and the incident photons will be trapped at the corrugated metal-organic interface. Actually, employment of dual metallic nanostructures consisted of metal NPs, and metallic grating is a promising approach to further enhance the performance of OSCs. $\mathrm{Li}$ et al. [122] incorporated Au NPs in the active layer and an Ag nanograting electrode as the back reflector to simultaneously excite the LSPR and SPP mode in the PBDTTT$\mathrm{CT}: \mathrm{PC}_{71} \mathrm{BM}$-based inverted OSCs. A broadband absorption enhancement and positive electrical effects are successfully achieved through the hybridized LPR (from the Au NPs) and SPR (from the Ag nanograting), which results in a significantly enhanced performance of the OSCs.

\subsection{Electrical performance}

Electromagnetic field enhancement induced by LSPR effect can result in the improved photon energy absorption and excess excitons production in the photoactive film [123-126]. Subsequently, the produced excitons interrelate with the plasmonic near-field of metal NPs to facilitate charge dissociation. The probability of exciton dissociation mainly depends on the internal electric field in OSCs, while the strong near-field induced by plasmonic NPs has been confirmed to enhance the probability [127]. Chen et al. [60] first proposed the performance improvement in OSCs by the integration of Au NPs into the hole transport layer (PEDOT:PSS) in 2009. An improvement of $20 \%$ in device performance was ascribed to the enhanced local electromagnetic field deriving from the near-field LSPR excitation. Furthermore, excitation of the LSPR induced by integration of Au NPs enhanced the probability in exciton generation and dissociation, which resulted in the noticeable improvement of the $J_{\mathrm{sc}}$ and the FF in OSCs. Later on, Wu et al. [108] employed Au NPs into buffer layer of OSCs and investigated their plasmonic effects on device performance. The rate of exciton generation drastically increased due to the enhancement in absorbed photons, which was ascribed to the LSPR modes and confirmed by the improved fluorescence intensity in steady-state photoluminescence (PL) spectroscopy. Besides, the probability of exciton dissociation of these devices enhanced from $79.2 \%$ to $84.4 \%$ with the addition of plasmonic NPs. Dynamic PL measurements indicated the reduced lifetime of photogenerated excitons, demonstrating the facilitated exciton dissociation due to the charge transfer process and plasmon-exciton coupling. Moreover, it is noteworthy 

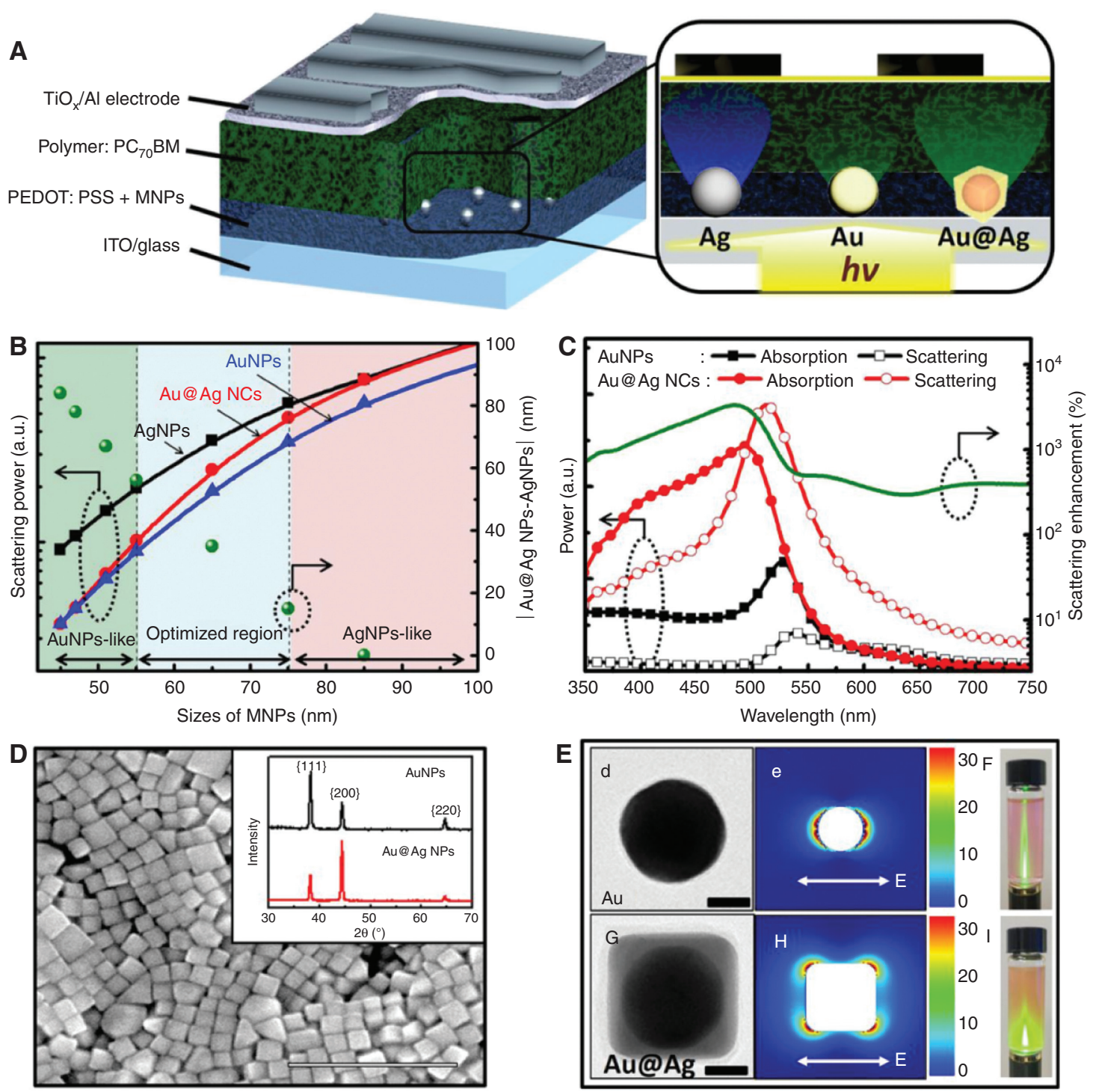

Figure 8: Schematic illustration, design and synthesis of plasmonic NPs.

(A) Schematic of a plasmonic OSC with the incorporation of metal NPs. (B-E) The scattering effect of the plasmonic metal NPs with core-shell structure. Reproduced with permission from Baek et al. [121]. Copyright 2014, American Chemical Society.

that the average lifetime of the photogenerated excitons decayed exponentially as the increased distance from the $\mathrm{Au}$ NPs surface, revealing the near-field essence of the enhanced exciton dissociation probability.

In addition to the noble metal NPs, other categories of metal NPs also have been demonstrated to enhance the probability of exciton dissociation. High-performance OSCs with incorporation of $\mathrm{Cu}$ NPs with size of $20 \mathrm{~nm}$ in P3HT layer were reported by Szeremeta et al. [77], and their optical and electrical properties were studied. It was confirmed that the enhanced efficiency did not rely solely on the light absorption improvement induced by plasmonic resonance, whereas $\mathrm{Cu}$ NPs played a substantial role in the increase of the exciton dissociation rate and photogeneration efficiency of charge carrier in active layer [77]. Liu et al. [76] embedded $\mathrm{Cu}$ NPs into the anodic buffer layer of PEDOT:PSS and improved device efficiency of OSCs based on P3HT and PTB7 (poly[[4,8-bis[(2-ethylhexyl) oxy]benzo[1,2-b:4,5-b0]dithiophene-2,6-diyl] [3-fluoro2-[(2-ethylhexyl)-carbonyl]thieno[3,4-b]thiophenediyl]]). In this case, the enhanced external quantum efficiency (EQE) and absorption can be ascribed to the enhanced saturation photocurrent density, related to the improvement in exciton dissociation rate.

The charge carriers produced by the exciton dissociation process should be transported and collected at the opposite electrodes for effective photocurrent generation and provide photocurrent direction. The charge carriers transport paths before collection have a significant effect on the bulk recombination and further electrical 
properties in OSCs, namely, $V_{\text {oc }}$ and FF. Actually, charge carriers hop between localized states is the usual method for charge transport [127]. In this section, we will discuss the influence of metal NPs on the charge transport and collection processes in OSCs. Wang et al. [128] reported positive effects due to the incorporation of Ag clusters formed by aggregation of Ag NPs with average size of $40 \mathrm{~nm}$ in PCDTBT:PC ${ }_{70} \mathrm{BM}$-based devices. The large-sized Ag clusters enhanced the values of $V_{\mathrm{oc}}, J_{\mathrm{sc}}, \mathrm{FF}$, and EQE in the OSCs, due to the enhanced light harvesting originating from the effective reflection and scattering of the aggregated Ag NPs. Besides, the enhanced charge transport and reduced resistance also contributed to the improvement of efficiency for the Ag NPs incorporated OSCs. Lee et al. [129] integrated Au NP-modified nitrogen (N)-doped or boron (B)-doped carbon nanotubes (CNTs) into OSCs to realize the multiple synergistic effects of exciton dissociation, charge generation, and transport enhancement. In consequence, a predominant PCE enhancement of the $\mathrm{PTB} 7: \mathrm{PC}_{70} \mathrm{BM}$-based device was obtained with the randomly distributed additives as shown in Figure 9.

Besides, the photocarriers mobility imbalance is commonly reported in OSCs, which induced the space charge limit for photocurrent [130, 131]. Sha et al. [132] propounded a plasmonic-electrical concept by incorporation of Ag NPs in different positions, which was employed to manipulate the recombination, transport, and collection of photocarriers via the redistribution of optical field in OSCs. The shortened transport path of the lower mobility photocarrier resulted in boosting the charge extraction and improving FF and $V_{\text {oc }}$. Ren et al. [133] proposed novel Au nanostars (Au NSs) incorporated between hole
A

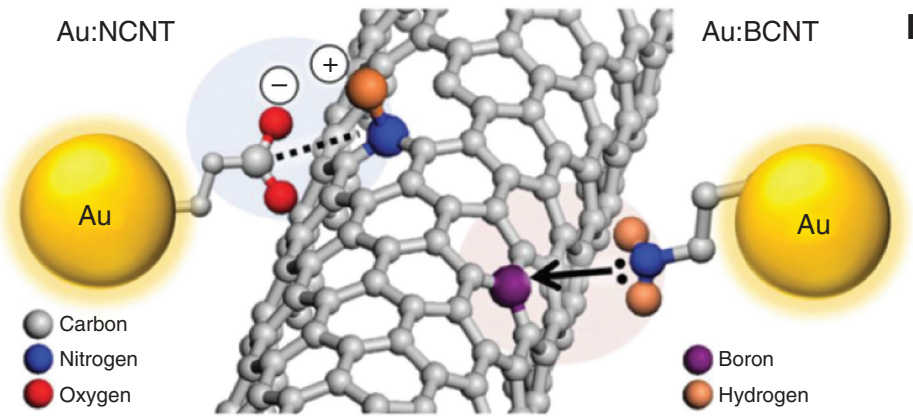

B
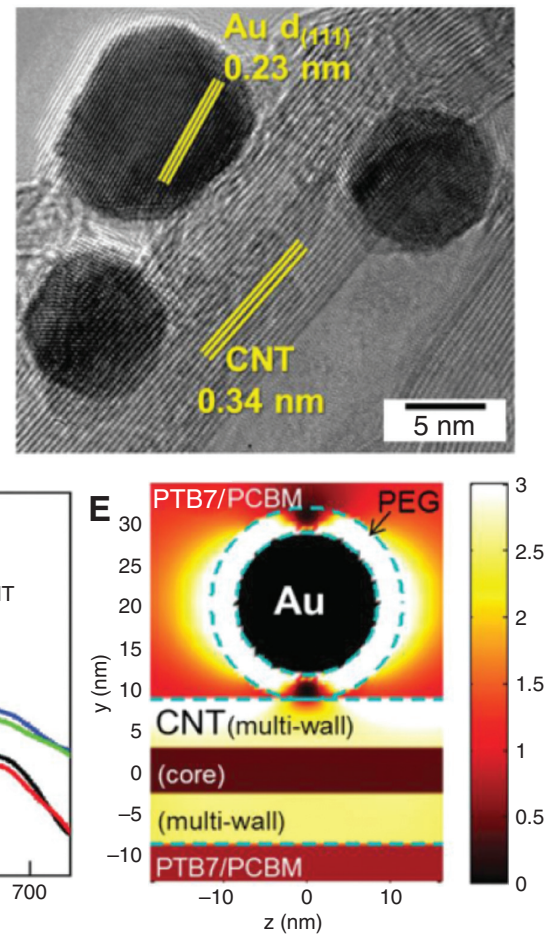
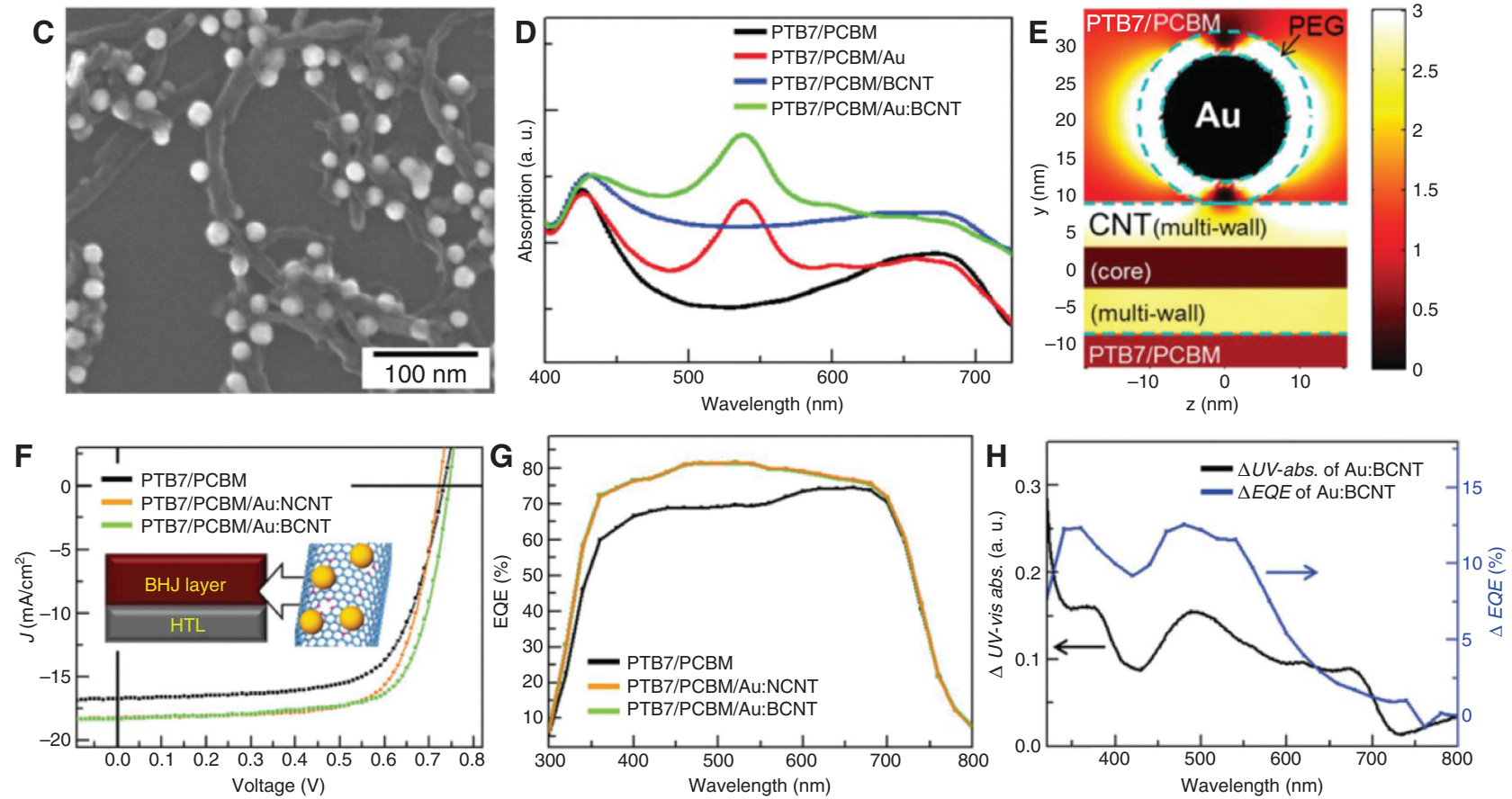

Figure 9: Illustration of Au NP-modified nitrogen or boron-doped carbon nanotubes (CNTs) into OSCs.

(A-E) Schematic diagram, surface morphology, and optical spectra of Au NP-modified N- or B-doped CNTs. (F-H) Corresponding device performance. Reproduced with permission from Lee et al. [129]. Copyright 2015, Wiley-VCH. 
transport layer and active layer. The excited asymmetric plasmonic modes enabled broadband absorption improvement in OSCs, rather than narrow spectral widths for regional enhancement. Simultaneously, the Au NSs could reduce the resistance of HTL and balance carrier through shortening the transport path length of relatively low-mobility holes. As a result, the PCE of the OSCs with the incorporation of Au NSs demonstrated a significant enhancement with both plasmon-optical and plasmonelectrical effects.

Morphology changes at the interface of the electrodes, buffer layer, and photoactive layer can also improve the carrier collection efficiency. The influence of embedding plasmonic metal NPs into the buffer layer of OSCs was dissimilar in contrast to the incorporated NPs in active layers. Choy et al. reported a 13\% enhancement of power conversion efficiency (PCE) in OSCs after the addition of PEGcapped Au NPs into the buffer layer of PEDOT:PSS, which primarily originated from the increased $J_{\mathrm{sc}}$ and FF [134]. The enhanced performance can be theoretically and experimentally ascribed to the increased interfacial roughness and enlarged interfacial area between PEDOT:PSS layer and P3HT:PCBM layer as well as increased PEDOT:PSS conductivity. The roughened interface has been demonstrate to benefit for the enhancement in hole collection efficiency. Fundamentally, it can be indicated that the device mechanisms of the minimal improvement from light absorption in the photoactive layer attributed to the lateral distribution feature of the near-field LSPR modes around $\mathrm{Au}$ NPs. These findings demonstrate that it is significantly imperative to delve into both optical and electrical characteristics for NPs employment to understand the device physics and optimize device performances.

The energy level differences among various functional materials in OSCs play an important role in carrier extraction. Therefore, adequate structure design to reduce the energy difference is crucial for high-performance OSCs. Particularly, the introduction of plasmonic metal NPs can effectively alter the local electrical field and change the function work of functional materials, leading to favorable band alignment. Cheng et al. [52] presented a high-performance 10-nm Au NP-embedded OSCs with enhanced $J_{\text {sc }}, V_{\text {oc }}$, and PCE by a factor of $8 \%, 9.5 \%$, and $22 \%$, respectively. Accordingly, they performed an in-depth investigation of the optimized performance after incorporation of Au NPs via impedance spectroscopy (IS) and valence band photoelectron spectra (VBPES), which can show the carrier dynamics and energy level changes. The IS results indicated that the improved photocurrent was induced by the suppression of electron-hole recombination and the enhancement in hole collection, as a consequence of the reduced contact resistance at the P3HT:PCBM/PEDOT:PSS interface. The VBPES results revealed a downshift of the Fermi energy level for the buffer layer (PEDOT:PSS) following the incorporation of Au NPs; thereby the energy barriers between the highest occupied molecular orbit level of P3HT and Fermi energy of PEOT:PSS were reduced, resulting in the enhanced collection efficiency. The barrier height and deletion width were also decreased at the interface, which improved the hole transport, arising from the lower contact resistance. Furthermore, the downshifted Fermi energy of PEDOT:PSS and the suppressed electronhole recombination led to the enhancement of $V_{\text {oc }}$. The variation of the energy level can be assigned to the hole concentration enhancement in PEDOT:PSS layer after the integration of high electron affiliative Au NPs.

Zhang et al. [135] integrated Au NPs into the electron transport layer of $\mathrm{TiO}_{2}$, resulting in the enhanced charge extraction by plasmonic-electrical effects in OSCs. As a consequence, the P3HT: $\mathrm{PC}_{61} \mathrm{BM}$-based OSCs achieved a peak PCE of $8.74 \%$. Besides, the $\mathrm{Au} \mathrm{NPs} / \mathrm{TiO}_{2}$-based OSCs worked within a plasmonic wavelength region of 560-600 nm, which was much longer than the absorbed wavelength of less than $400 \mathrm{~nm}$ for the $\mathrm{TiO}_{2}$-based control devices. It was confirmed that the charge transfer of excited carriers from plasmonic $\mathrm{NPs}$ to $\mathrm{TiO}_{2}$ and trap filling in $\mathrm{TiO}_{2}$ films was responsible for these improvements in OSCs, rather than the induced field effect at the $\mathrm{TiO}_{2}$ surface as shown in Figure 10. The device performance with incorporation of plasmonic metal NPs in OSCs is demonstrated in Table 1.

\subsection{Device stability}

A novel perspective on the function of metal NPs incorporated into the photoactive layers has been reported in recent years to improve the structural stability, causing reduced device degradation rate of OSCs under prolonged solar illumination. In this case, plasmonic NPs can function as stabilizers to impede the singlet oxygen and quench the triplet excitons to avoid the photo-oxidation process. This property can elucidate the difference of the enhanced absorption and IPCE in OSCs. Paci et al. [78] embedded Ag NPs into the P3HT:PCBM layer to enhance the morphological and structural properties of OSCs. The devices showed a superior photovoltaic performance with mitigated degradation rate after continuous irradiation for a long period. Overall, the employment of metal NPs could result in a plasmon-mediated absorption enhancement to improve the device efficiency, as well as an increased structural stability to benefit the 


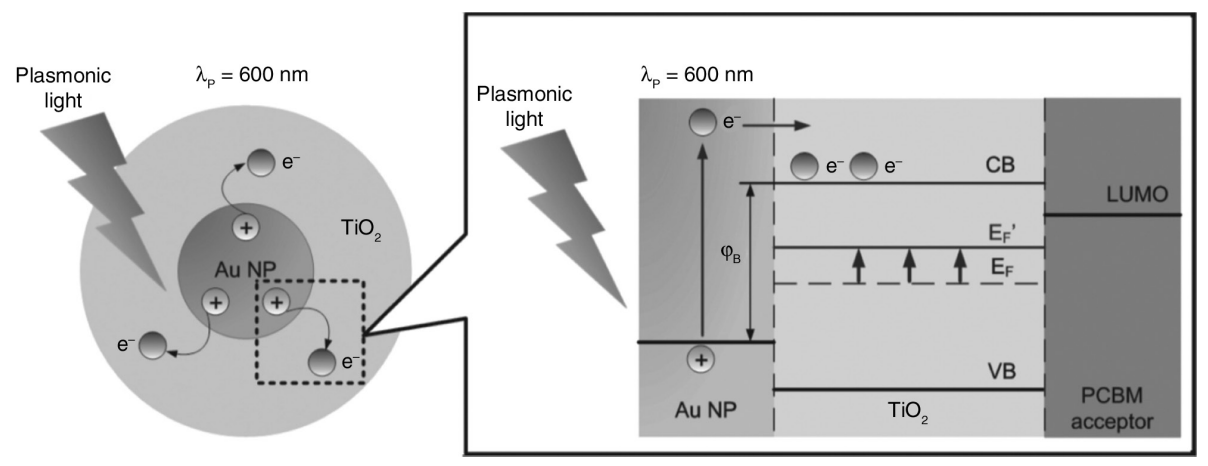

Figure 10: Diagram of plasmonic metal NP-induced charge injection process.

Electrons are extracted from PCBM layer to $\mathrm{TiO}_{2}$ layer in OSCs. Reproduced with permission from Zhang et al. [135]. Copyright 2013, Wiley-VCH.

device durability. Subsequently, the same group utilized surfactant-free $\mathrm{Au}$ NPs into the photoactive layer and obtained improved photo and thermal stability in OSCs. Figure 11 demonstrates the schematic of the photo-oxidation process and the role of Au NPs acting as triplet quenchers [136]. Kakavelakis et al. [137] employed laserablated Al NPs to achieve highly efficient and stable OSCs. An efficiency enhancement of $30 \%$ was obtained due to the multiple scattering effects, as well as the long operation time of $\sim 150$ hours induced by the plasmonic Al NPs.

\section{Plasmonics in PeSCs}

Perovskite materials have gained tractions for solar cells in recent decade due to their high absorption coefficient, low exciton binding energy, wide absorption wavelength, high bipolar charge mobility, and long photocarrier diffusion length. Basically, PeSCs permit relatively thicker photoactive film than OSCs for more reliable light harvesting; hence, less light can escape once it enters into the absorber. Moreover, PeSCs are solution processed, combining high performance with cost-effective techniques. Meanwhile, the tunable bandgap and high color purity, with reasonable ionization energy (IE) comparable to that of common hole-injection materials, enable perovskite attractive emitting materials applied in light-emitting diodes (LEDs) [138-140]. Perovskite can function as both a good emitter and a good absorber, which makes it unique among common semiconductors employed in solar cells and LEDs.

So far, the PCEs of PeSCs have undergone a rapid growth over the past few years, with the state-of-the-art value of $25.2 \%$ certified by NREL [https://www.nrel.gov/ pv/cell-efficiency.html], which are comparable with the commercial Si solar cells. Although perovskite films have been already efficient light harvesters for past years, yet in fact, the obtained PCEs of PeSCs are still below the Shockley-Queisser limit, requiring new methods to further improve their absorption [40, 141, 142]. In fundamental, wavelength-dependent light distribution in devices renders the not-enough broadband light absorption in PeSCs. Specifically, photon capture ability is not high within the IR frequencies because of the rapid decays of extinction coefficient, which influences considerably the conversion efficiency from incident photon to electron. Besides, because of the high refractive indices of perovskite, the inner interface reflection of PeSCs is stronger than OSCs, which leads to the unanticipated light losses in devices. Besides the technical reason, minimal thickness of the perovskite layer should be pursued to reduce the lead content under the consideration of the potential ecotoxicological issues, which causes a dilemma for the effective light absorption. Therefore, optimization of the optical design of the PeSCs should be explored to address this issue to reach a determined performance.

Incorporation of plasmonic metal NPs is a realistic way to enhance light absorption without changing the device configuration. Zhang et al. initially employed the core-shell metal-dielectric structure ( $\left.\mathrm{Au} @ \mathrm{SiO}_{2} \mathrm{NPs}\right)$ in photoactive layer to improve the PCE of organometal halide PeSC to $11.4 \%$ on average, whereas the control device is only $10.7 \%$ [63]. The enhanced performance could be attributed to the reduced exciton binding energy and the improved charge carrier generation after the inclusion of metal NPs, rather than the light absorption enhancement, confirmed by the time-resolved and steadystate temperature-dependent PL measurement. Although the physically fundamental origin of the improvement in PeSCs was unknown, this confirmed that plasmonic NPs could be utilized to improve the device performance. Subsequently, the same group adopted core-shell Ag@ $\mathrm{TiO}_{2}$ 
Table 1: Device performance with incorporation of plasmonic metal NPs in OSCs.

\begin{tabular}{|c|c|c|c|c|c|c|c|c|}
\hline $\begin{array}{l}\text { Absorber } \\
\text { materials }\end{array}$ & Plasmonic NPs & Position of NPs & $J_{s c}\left(\mathrm{~mA} / \mathrm{cm}^{2}\right)$ & $V_{\text {oc }}(\mathrm{V})$ & FF (\%) & PCE (\%) & Mechanism & Refs. \\
\hline Р3НТ:РCBM & Au NPs & Active layer & $9.77(8.27)$ & $0.6(0.6)$ & $63.38(53.22)$ & $3.71(2.64)$ & $\begin{array}{l}\text { LSPR } \\
\text { Scattering }\end{array}$ & [92] \\
\hline Р3НT:РCBM & PEG-Au NPs & $\begin{array}{l}\text { Active layer } \\
\text { Buffer layer }\end{array}$ & $9.74(8.35)$ & $0.61(0.61)$ & $65.00(61.92)$ & $3.85(3.16)$ & LSPR & [107] \\
\hline Р3НT:РCBM & Au NPs & Buffer layer & $10.22(9,16)$ & $0.59(0.59)$ & $70.32(66.06)$ & $4.24(3.57)$ & $\begin{array}{l}\text { LSPR } \\
\text { Enhanced exciton } \\
\text { dissociation }\end{array}$ & [108] \\
\hline PTCBI & Ag NPs & $\begin{array}{l}\text { Interface of } \\
\text { tandem device }\end{array}$ & $4.90(4.25)$ & $0.93(0.47)$ & $55(55)$ & $2.5(1.1)$ & LSPR & [111] \\
\hline P3HT:PCBM & $\begin{array}{l}\text { Au-Ag alloy } \\
\text { NPs }\end{array}$ & $\begin{array}{l}\text { Electrode/buffer } \\
\text { layer interface }\end{array}$ & $8.74(7.37)$ & $0.62(0.62)$ & $55(55)$ & $3.03(2.54)$ & LSPR & [100] \\
\hline PCDTBT:PC $\mathrm{P}_{71} \mathrm{BM}$ & Ag NPs & Active layer & $12.12(11.63)$ & $0.87(0.90)$ & $61(57)$ & $6.4(5.9)$ & Scattering & [119] \\
\hline P3HT:PCBM & Ag NPs & $\begin{array}{l}\text { Electrode/buffer } \\
\text { layer interface }\end{array}$ & $9.33(7.89)$ & $0.58(0.59)$ & $53(52)$ & $2.82(2.41)$ & $\begin{array}{l}\text { Scattering } \\
\text { Resistance } \\
\text { reduction }\end{array}$ & [120] \\
\hline Р3HT:РCBM & Ag NPs & $\begin{array}{l}\text { Electrode/active } \\
\text { layer interface }\end{array}$ & $9.9(7.89)$ & $0.57(0.59)$ & $48(52)$ & $3.2(2.41)$ & $\begin{array}{l}\text { Scattering } \\
\text { Resistance } \\
\text { reduction }\end{array}$ & [120] \\
\hline PCDTBT:PC 70 BM & Au@Ag NCs & Buffer layer & $11.10(10.32)$ & $0.87(0.87)$ & $63(58)$ & $6.08(5.21)$ & Scattering & [121] \\
\hline PTB7:PC 70 BM & Au@Ag NCs & Buffer layer & $17.38(16.43)$ & $0.74(0.74)$ & $68(64)$ & $8.74(7.78)$ & Scattering & [121] \\
\hline PBDTTT-CT: & Au NPs & Active layer & 18.39 (17.09) & $0.76(0.76)$ & $62.87(58.43)$ & $8.79(7.59)$ & LSPR & [122] \\
\hline $\mathrm{PC}_{71} \mathrm{BM}$ & Ag nanograting & Electrode & & & & & SPP & \\
\hline PIDTT- & Ag@TiO_@Pa & Active layer & $14.13(12.66)$ & $0.98(0.96)$ & $65(62)$ & $9.03(7.54)$ & Scattering & [101] \\
\hline DFBT:PC ${ }_{71} B M$ & NPs & & & & & & $\begin{array}{l}\text { Enhanced exciton } \\
\text { generation }\end{array}$ & \\
\hline PTB7- & Ag@TiO_@Pa & Active layer & $18.05(16.32)$ & $0.81(0.80)$ & $73(71)$ & $10.72(9.28)$ & Scattering & [101] \\
\hline Th:PC ${ }_{71} B M$ & NPs & & & & & & $\begin{array}{l}\text { Enhanced exciton } \\
\text { generation }\end{array}$ & \\
\hline P3HT:IC ${ }_{60} \mathrm{BA}$ & $\begin{array}{l}\mathrm{Ag} @ \mathrm{TiO}_{2} @ \mathrm{~Pa} \\
\mathrm{NPs}\end{array}$ & Active layer & $11.96(10.56)$ & $0.85(0.83)$ & $70(67)$ & $7.10(5.89)$ & $\begin{array}{l}\text { Scattering } \\
\text { Enhanced exciton } \\
\text { generation }\end{array}$ & [101] \\
\hline PTB7- & Ag@TiO_@Pa & Active layer & $21.90(20.70)$ & $0.78(0.77)$ & $73(71)$ & $12.51(13.01)$ & Scattering & [101] \\
\hline Th:BTR:PC ${ }_{71} B M$ & NPs & & & & & & $\begin{array}{l}\text { Enhanced exciton } \\
\text { generation }\end{array}$ & \\
\hline Р3НТ:РCBM & Au NPs & Buffer layer & $10.18(8.95)$ & $0.59(0.59)$ & $69.8(65.9)$ & $4.19(3.48)$ & $\begin{array}{l}\text { Enhanced exciton } \\
\text { dissociation }\end{array}$ & [60] \\
\hline PTB7:PC 70 BM & Cu NPs & Buffer layer & $17.10(16.01)$ & $0.73(0.72)$ & $59.8(59.3)$ & $7.43(6.79)$ & $\begin{array}{l}\text { Enhanced exciton } \\
\text { dissociation }\end{array}$ & [76] \\
\hline PCDTBT:PC ${ }_{70} B M$ & Ag NPs & Active layer & $11.61(10.79)$ & $0.86(0.86)$ & $69(68)$ & $7.1(6.3)$ & $\begin{array}{l}\text { Scattering } \\
\text { Enhanced charge } \\
\text { transport }\end{array}$ & [128] \\
\hline PTB7:PC ${ }_{70}$ BM & Au NPs:NCNT & Active layer & $18.21(16.71)$ & $0.72(0.73)$ & $71.78(68.03)$ & $9.24(8.12)$ & $\begin{array}{l}\text { Enhanced exciton } \\
\text { dissociation } \\
\text { Charge transport }\end{array}$ & [129] \\
\hline PTB7:PC 70 BM & Au NPs:NCNT & $\begin{array}{l}\text { Active layer } \\
\text { Buffer layer }\end{array}$ & $18.50(16.71)$ & $0.74(0.73)$ & $72.61(68.03)$ & $9.75(8.12)$ & $\begin{array}{l}\text { Enhanced exciton } \\
\text { dissociation } \\
\text { Charge transport }\end{array}$ & [129] \\
\hline $\begin{array}{l}\text { PBDT- } \\
\text { TS1:PC }{ }_{71} B M\end{array}$ & Au NSs & $\begin{array}{l}\text { Buffer layer/ } \\
\text { active layer } \\
\text { interface }\end{array}$ & $18.72(17.08)$ & $0.81(0.81)$ & $67.66(66.89)$ & $10.25(9.26)$ & $\begin{array}{l}\text { Broadband } \\
\text { enhancement } \\
\text { Balanced charge } \\
\text { collection }\end{array}$ & [133] \\
\hline Р3НТ:РCBM & PEG-Au NPs & Buffer layer & $8.94(8.5)$ & $0.63(0.62)$ & $62(58)$ & $3.51(3.1)$ & $\begin{array}{l}\text { LSPR } \\
\text { Charge collection }\end{array}$ & [134] \\
\hline
\end{tabular}


Table 1 (continued)

\begin{tabular}{|c|c|c|c|c|c|c|c|c|}
\hline $\begin{array}{l}\text { Absorber } \\
\text { materials }\end{array}$ & Plasmonic NPs & Position of NPs & $J_{s c}\left(\mathrm{~mA} / \mathrm{cm}^{2}\right)$ & $V_{o c}(V)$ & FF (\%) & PCE (\%) & Mechanism & Refs. \\
\hline PTB7:PC 71 BM & Au NPs & Buffer layer & $18.07(17.23)$ & $0.71(0.71)$ & $68.1(65.5)$ & $8.74(8.02)$ & $\begin{array}{l}\text { Enhanced charge } \\
\text { extraction }\end{array}$ & [135] \\
\hline РЗНТ:РCBM & Ag NPs & Active layer & $7.03(4.78)$ & $0.61(0.55)$ & $48(40)$ & $2.06(1.05)$ & $\begin{array}{l}\text { Enhanced device } \\
\text { stability }\end{array}$ & [136] \\
\hline Р3НТ:РCBM & Al NPs & Active layer & $11.31(8.59)$ & $0.6(0.6)$ & $59(61)$ & $4.00(3.14)$ & $\begin{array}{l}\text { Scattering } \\
\text { Enhanced device } \\
\text { stability }\end{array}$ & [137] \\
\hline
\end{tabular}

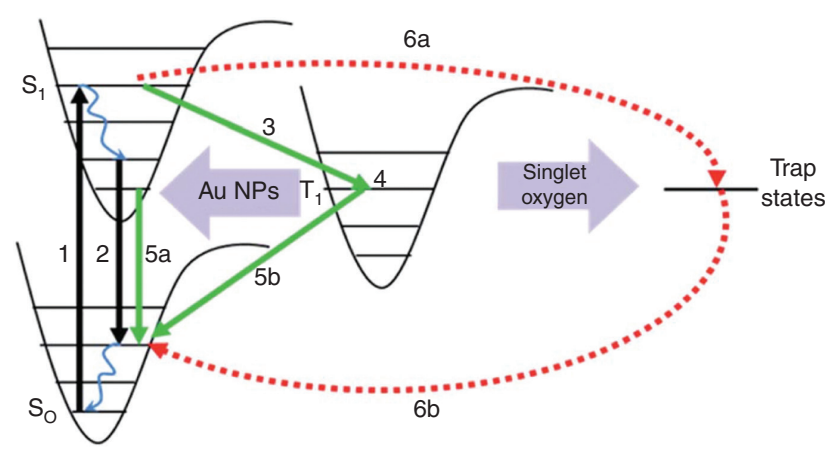

Figure 11: Schematic of the photo-oxidation process in the $\mathrm{Au}$ $\mathrm{NP}-$-embedded active layer.

Reproduced with permission from Paci et al. [136]. Copyright 2012, The Royal Society of Chemistry.

NPs to boost the peak efficiency of the PeSCs to $16.3 \%$. The device demonstrated a systematic enhancement in photocurrent; however, enhanced light harvest was not considered as the main reason for this enhancement [143]. Indeed, highly polarizable NPs effectively improved the radiative decay of excitons and the reabsorption of emitted light, corresponding to an innovative photon recycling mode. This study illuminated the subtle interactions between the radiative dipoles of the excitons in the perovskite material and the dipoles of the plasmonic NPs. Such plasmonic and photonic schemes can facilitate the performance of PeSCs to the Shockley-Queisser limit (theoretical limit). More recently, Yao et al. [101] proposed a designed core-bishell structure of $\mathrm{Ag} / \mathrm{TiO}_{2} / \mathrm{Pa}$ (benzoic acid-fullerene) NPs, enabling their compatibility with perovskite absorbers. Furthermore, the $\mathrm{TiO}_{2} / \mathrm{Pa}$ bishell with appropriate energy alignment could trigger efficient plasmon-exciton coupling, thus promoting exciton dissociation and charge carrier collection. The enhanced light absorption and charge extraction of PeSCs with the $\mathrm{Ag} / \mathrm{TiO}_{2} / \mathrm{Pa}$ NPs contributed to the enhanced $J_{\text {sc }}$ and FF, respectively (Figure 12). In consequence, the device performance with the inclusion of $\mathrm{Ag} / \mathrm{TiO}_{2} / \mathrm{Pa} \mathrm{NPs}$ presented an improvement by $10.2 \%$ from $18.4 \%$ to $20.2 \%$.

Metal NPs can also be added into the buffer layers of PeSCs, demonstrating different mechanisms for the improvement of light harvesting without immediate contact with the perovskite semiconductors [144]. Lee et al. dispersed Au NPs with an average diameter of $15 \mathrm{~nm}$ into the hole transport layer of spiro-OMeTAD, eliminating the demand for an insulating shell [145]. The inclusion of $\mathrm{Au}$ NPs resulted in only a slight improvement in PCE, from $12.66 \%$ for the control device to $12.74 \%$. The improved EQE profile was a combined response of a slight enhancement in $J_{\mathrm{sc}}$, remained $\mathrm{FF}$, and a slightly decreased $V_{\text {oc }}$ after the incorporation of Au NPs. In this case, Au NPs were reported to aggregate near the spiroOMeTAD/perovskite interface, rather than dispersed uniformly throughout the spiro-OMeTAD layer, which induced an unexpected energetic barrier at the interface and led to the slightly reduced $V_{\text {oc }}$. In fundamental, the boosted device performance was ascribed to the absorption improvement and decreased resistance of the spiroOMeTAD buffer layer.

As mentioned before, the perovskite film presented poor absorption in the IR frequency, influencing the conversion efficiency from photon to electron. Incorporation of plasmonic NPs has been investigated to be an efficient way to circumvent this issue. Yue et al. [146] demonstrated that the employment of optimized closely spaced Ag NP array could cause an IR-band absorption enhancement of $58.2 \%$ through a full-wave simulation method (Figure 13). Here, plasmonic NPs were reported to benefit for the production of hotspots in the perovskite films, significantly enhancing their localized light absorption due to the nonlinear absorption mechanisms and light-flow circulation. $\mathrm{Lu}$ et al. reported plasmonic-enhanced PeSCs with inclusion of irregular alloy $\mathrm{Au}-\mathrm{Ag}$ popcorn NPs into the mesoporous layer [64]. This device presented broadband 
A

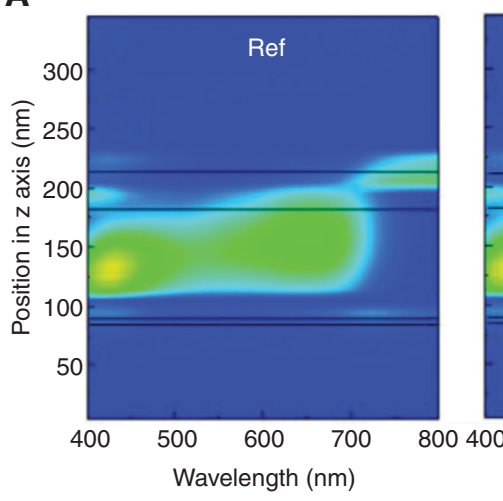

B

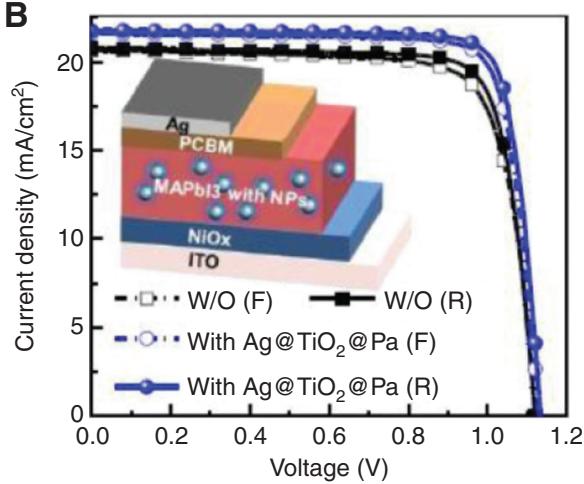

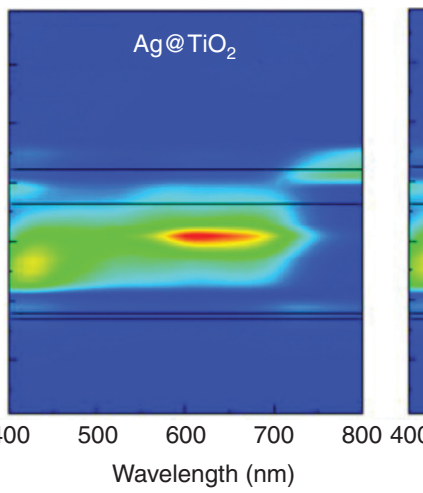

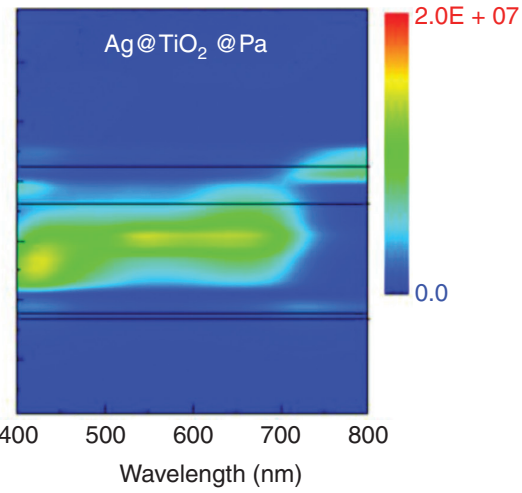

C

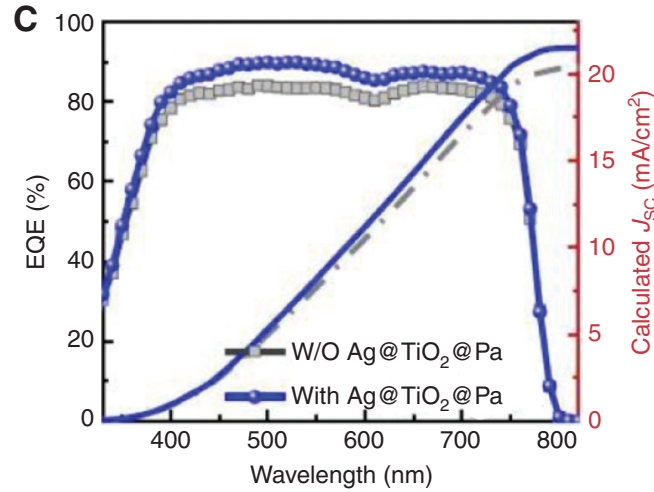

Figure 12: Performance characterizations of plasmonic devices.

(A) Absorptive power distribution of device with adding of Ag@TiO, or Ag@TiO,@Pa compared to the reference OSC. (B, C) Current density and EQE performance with and without the incorporation of NPs. Reproduced with permission from Yao et al. [101]. Copyright 2019, American Chemical Society.

optical absorption enhancement from UV to near-IR wavelength range induced by the integrated NPs. Furthermore, the plasmonic NPs could also induce increased charge transfer at the $\mathrm{TiO}_{2} /$ perovskite interface, resulting in an ultimate PCE enhancement of $15.7 \%$, from $8.9 \%$ to $10.3 \%$.

As the potential toxicity issues related to lead [147], commercialization of PeSCs needs the lowest possible thickness of the perovskite films to decrease the lead content. However, insufficient light absorption could occur in this case due to the ultrathin active layers. Employment of plasmonic NPs has been confirmed to be an effective way to address this dilemma. Cai et al. [148] proposed a novel nanoplasmonic lumpy structure consisting of a large Ag NP at the core and smaller Ag NPs at the surrounding, which was integrated at the rear/front surfaces of the perovskite films. The lumpy Ag NP-induced LSPR effects and light scattering led to the enhanced photocurrent of a PeSC with 50-nm absorber, which is higher than the flat control device with 300-nm absorber. Interestingly, when the thickness of perovskite layer reduced to $10 \mathrm{~nm}$, photocurrent was predicted to show a fourfold enhancement. This report represents an important step in the field of high-performance thin-absorber lead-based or lead-free PeSCs. The device performance with incorporation of plasmonic metal NPs in PeSCs is summarized in Table 2.

So far, the reported works have mainly focused on the enhanced light harvesting by incorporation of plasmonic NPs in single-junction PeSCs. However, perovskite/ crystalline Si tandem solar cells are hotly pursued nowadays due to the easy adjustment of bandgap in perovskite materials and their low nonradiative recombination rates, which can further enable substantial improvements in PCE $[149,150]$. Thus, appreciated design of the tandem device to improve the device optics with plasmonic NPs coupling can be an effective approach to achieve more efficient PeSCs in future.

\section{Conclusions and outlook}

The cost-effective plasmonic metal NP-based solutionprocessed OSCs and PeSCs represent an important stride to the prominently enhanced device efficiency and stability. Fundamentally, plasmonic NPs can be facilely incorporated into either the photoactive or the buffer layers or at interfaces for enhanced optical absorption due to LSPR 


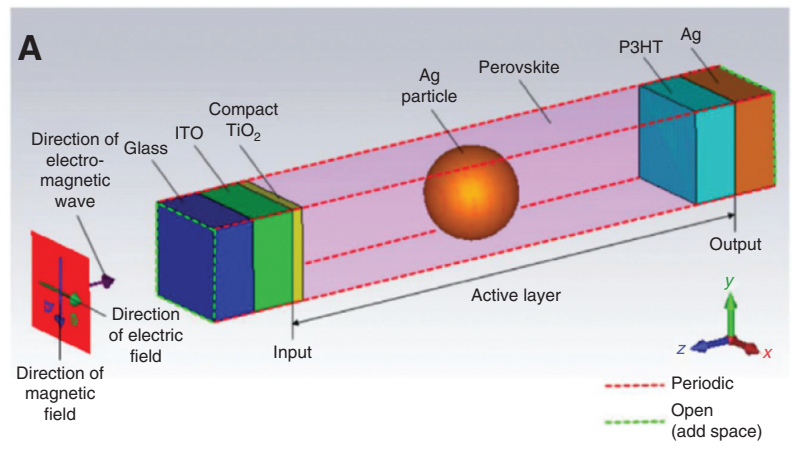

B

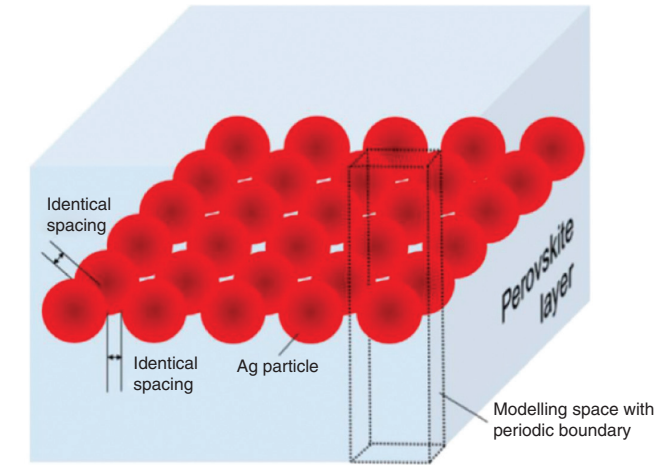

Figure 13: Model of multiple particle array-incorporated PeSCs. (A) Model of sandwiched PeSC. (B) Diagram of simulated design of multiple particle arrays embedded in perovskite layer. Reproduced with permission from Yue et al. [146]. Copyright 2016, Elsevier.

and light scattering effect and enhanced electrical properties in exciton dissociation, charge transport, and collection of the OSCs and PeSCs, without compromising the device architecture. Careful consideration should be given on the fabrication methods of NPs with controlled parameters, while theoretical simulation has been reported to provide supportive guidelines for their designs. A thorough investigation of the underlying enhancement mechanisms relating to interrelationships between the plasmonic NPs parameters including shape, size, distribution, and surrounding medium with the device performance characteristics is studied and highlighted. Moreover, enhanced photodegradation stability is also observed via the NP incorporation into the photoactive layer.

To date, the reported works have demonstrated the improvements in device performance for OCSs and PeSCs arising from the enhanced $J_{\mathrm{sc}}, V_{\mathrm{oc}}$, and FF and present a wide range of in-depth enhancement mechanisms. For PeSCs, it is still unknown whether the plasmonic light trapping is the foremost factor for the reduction of optical losses. Therefore, more researches are required to clarify the absorption increase of plasmonic NPs with respect to their potential in electrical properties enhancement for OSCs and PeSCs. Besides, efforts to address the stability issue with plasmonic

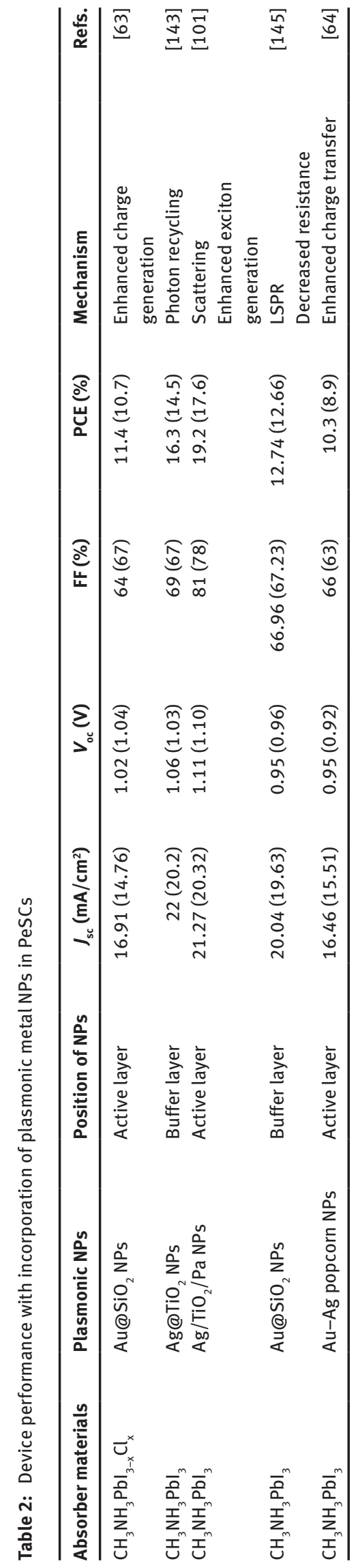


NP incorporation in various layers of OSCs and PeSCs should be pursued. Furthermore, reduction of the manufacturing cost for such systems with large scale should be further investigated to commercialize applications. We hope this work will accelerate new insights and further investigations to boost the development of plasmonic metal NP-based OSCs and PeSCs, especially for future commercialized applications.

Acknowledgments: This work was supported by the National Key Research and Development Program of China and the National Natural Science Foundation of China (grants 2017YFB0404500, 61590930, 61825402, 61675085, and 61705075).

\section{References}

[1] Chou CH, Chen FC. Plasmonic nanostructures for light trapping in organic photovoltaic devices. Nanoscale 2014;6:8444-58.

[2] Wright M, Uddin A. Organic-inorganic hybrid solar cells: a comparative review. Sol Energy Mater Sol Cells 2012;107: 87-111.

[3] Stratakis E, Kymakis E. Nanoparticle-based plasmonic organic photovoltaic devices. Mater Today 2013;16:133-46.

[4] Chan K, Wright M, Elumalai N, Uddin A, Pillai S. Plasmonics in organic and perovskite solar cells: optical and electrical effects. Adv Optical Mater 2017;5:1600698.

[5] Bi Y-G, Feng J, Ji J-H, et al. Nanostructures induced light harvesting enhancement in organic photovoltaics. Nanophotonics 2018;7:371-91.

[6] Chen J-D, Jin T-Y, Li Y-Q, Tang J-X. Recent progress of light manipulation strategies in organic and perovskite solar cells. Nanoscale 2019;11:18517-36.

[7] Wang L, Wang H-Y, Wei H-T, et al. Unraveling charge separation and transport mechanism in aqueous-processed polymer/ CdTe nanocrystal hybrid solar cells. Adv Energy Mater 2014;4:1308812.

[8] Branker K, Pathak MJM, Pearce JM. A review of solar photovoltaic levelized cost of electricity. Renew. Sustain. Energy Rev. 2011;15:4470-82.

[9] Krebs FC. Fabrication and processing of polymer solar cells: a review of printing and coating techniques. Sol Energy Mater Sol Cells 2009;93:394-412.

[10] Søndergaard RR, Hösel M, Krebs FC. Roll-to-roll fabrication of large area functional organic materials. J Polym Sci B 2013;51:16-34.

[11] Krebs FC, Nielsen TD, Fyenbo J, Wadstrø M, Pedersen MS. Manufacture, integration and demonstration of polymer solar cells in a lamp for the "Lighting Africa" initiative. Energy Environ Sci 2010;3:512-25.

[12] Krebs FC, Espinosa N, Hösel M, Søndergaard RR, Jørgensen M. Rise to power-OPV-based solar parks. Adv Mater 2014;26:29-39.

[13] Ren X, Li X, Choy WCH. Optically enhanced semi-transparent organic solar cells through hybrid metal/nanoparticle/dielectric nanostructure. Nano Energy 2015;17:187-95.
[14] Ou X-L, Xu M, Feng J, Sun H-B. Flexible and efficient ITO-free semitransparent perovskite solar cells. Sol Energy Mater Sol Cells 2016;157:660-5.

[15] Xu M, Feng J, Ou X-L, et al. Surface passivation of perovskite film by small molecule infiltration for improved efficiency of perovskite solar cells. IEEE Photon J 2016;8:6804807.

[16] Xu M, Feng J, Fan Z-J, et al. Flexible perovskite solar cells with ultrathin Au anode and vapour-deposited perovskite film. Sol Energy Mater Sol Cells 2017;169:8-12.

[17] Ma R, Feng J, Yin D, Sun H-B. Highly efficient and mechanically robust stretchable polymer solar cells with random buckling. Org Electron 2017;43:77-81.

[18] Yin D, Jiang N-R, Liu Y-F, et al. Mechanically robust stretchable organic optoelectronic devices built using a simple and universal stencil-pattern transferring technology. Light Sci Appl 2018;7:35.

[19] Lu H, Ren X, Ouyang D, Choy WCH. Emerging novel metal electrodes for photovoltaic applications. Small 2018;14:1703140.

[20] Min H, Kim M, Lee S-U, et al. Efficient, stable solar cells by using inherent bandgap of a-phase formamidinium lead iodide. Science 2019;366:749-53.

[21] Kang SB, Kim J-H, Jeong MH, et al. Stretchable and colorlesS freestanding microwire arrays for transparent solar cells with flexibility. Light Sci Appl 2019;8:121.

[22] Ren H, Ren X, Huang Z, Wu X. Synergetic light trapping effects in organic solar cells with a patterned semi-transparent electrode. Phys Chem Chem Phys 2019;21:11306-12.

[23] Forrest SR. The path to ubiquitous and low-cost organic electronic appliances on plastic. Nature 2004;428:911-8.

[24] Park SH, Roy A, Beaupré S, et al. Bulk heterojunction solar cells with internal quantum efficiency approaching $100 \%$. Nat Photonics 2009;3:297-302.

[25] Kaltenbrunner M, White MS, Głowacki ED, et al. Ultrathin and lightweight organic solar cells with high flexibility. Nat Commun 2012;3:1-7.

[26] Liu Z, Li J, Yan F. Package-free flexible organic solar cells with graphene top electrodes. Adv Mater 2013;25:4296-301.

[27] Yuan J, Zhang Y, Zhou L, et al. Single-junction organic solar cell with over $15 \%$ efficiency using fused-ring acceptor with electron-deficient core. Joule 2019;3:1140-51.

[28] Dong S, Zhang K, Jia T, et al. Suppressing the excessive aggregation of nonfullerene acceptor in blade-coated active layer by using $\mathrm{n}$-type polymer additive to achieve large-area printed organic solar cells with efficiency over $15 \%$. EcoMat 2019;1:e12006.

[29] Han TH, Lee JW, Choi C, et al. Perovskite-polymer composite cross-linker approach for highly-stable and efficient perovskite solar cells. Nat Commun 2019;10:1-10.

[30] Jiang Q, Zhao Y, Zhang X, et al. Surface passivation of perovskite film for efficient solar cells. Nat Photonics 2019;13:460-6.

[31] Bi E, Tang W, Chen H, et al. Efficient perovskite solar cell modules with high stability enabled by iodide diffusion barriers. Joule 2019;3:2748-60.

[32] Meng L, Zhang Y, Wan X, et al. Organic and solution-processed tandem solar cells with $17.3 \%$ efficiency. Science 2018;361:1094-8.

[33] Pillai S, Green MA. Plasmonics for photovoltaic applications. Sol Energy Mater Sol Cells 2010;94:1481-6.

[34] Longeaud C, Allah AF, Schmidt J, Yaakoubi ME, Berson S, Lemaitre N. Determination of diffusion lengths in organic semiconductors: correlation with solar cell performances. Org Electron 2016;31:253-7. 
[35] Yu G, Gao J, Hummelen JC, Wudl F, Heeger AJ. Polymer photovoltaic cells: enhanced efficiencies via a network of internal donor-acceptor heterojunctions. Science 1995;270:1789-91.

[36] Ma W, Yang C, Gong X, Lee K, Heeger AJ. Thermally stable, efficient polymer solar cells with nanoscale control of the interpenetrating network morphology. Adv Funct Mater 2005;15:1617.

[37] Tang Z, Tress W, Inganäs O. Light trapping in thin film organic solar cells. Mater Today 2014;17:389-96.

[38] Choy WCH, Chan WK, Yuan Y. Recent advances in transition metal complexes and light-management engineering in organic optoelectronic devices. Adv Mater 2014;26:5368-99.

[39] Stranks SD, Eperon GE, Grancini G, et al. Electron-hole diffusion lengths exceeding 1 micrometer in an organometal trihalide perovskite absorber. Science 2013;342:341-4.

[40] Ahn N, Son DY, Jang IH, Kang SM, Choi M, Park NG. Highly reproducible perovskite solar cells with average efficiency of $18.3 \%$ and best efficiency of $19.7 \%$ fabricated via Lewis base adduct of lead (II) iodide. J Am Chem Soc 2015;137:8696-9.

[41] Rockstuhl C, Fahr S, Lederer F. Absorption enhancement in solar cells by localized plasmon polaritons. J Appl Phys 2008;104:123102.

[42] Atwater HA, Polman A. Plasmonics for improved photovoltaic devices. Nat Mater 2010;9:205-13.

[43] Yu ET, Lagemaat JVD. Photon management for photovoltaics. MRS Bull 2011;36:424-8.

[44] Qiao L, Wang D, Zuo L, et al. Localized surface plasmon resonance enhanced organic solar cell with gold nanospheres. Appl Energ 2011;88:848-52.

[45] Cheng PP, Ma GF, Li J, et al. Plasmonic back scattering enhancement for inverted polymer solar cells. J Mater Chem 2012;22:22781-7.

[46] Notarianni M, Vernon K, Chou A, Aljada M, Liu J, Motta N. Plasmonic effect of gold nanoparticles in organic solar cells. Sol Energy 2014;106:23-37.

[47] Carretero-Palacios S, Jiménez-Solano A, Míguez H. Plasmonic nanoparticles as light-harvesting enhancers in perovskite solar cells: a user's guide. ACS Energy Lett 2016;1:323-31.

[48] Lee JY, Peumans P. The origin of enhanced optical absorption in solar cells with metal nanoparticles embedded in the active layer. Opt Express 2010;18:10078-87.

[49] Wang H, Wang H-Y, Chen Q-D, et al. Hybrid-state dynamics of dye molecules and surface plasmon polaritons under ultrastrong coupling regime. Laser Photon Rev 2018;12:1700176.

[50] Dubi Y, Sivan Y. "Hot” electrons in metallic nanostructures-nonthermal carriers or heating? Light Sci Appl 2019;8:89.

[51] Wu B, Wu X, Guan C, et al. Uncovering loss mechanisms in silver nanoparticle-blended plasmonic organic solar cells. Nat Commun 2013;4:2004.

[52] Cheng CE, Pei Z, Hsu CC, Chang CS, Chien FS-S. Hole transit in P3HT: PCBM solar cells with embedded gold nanoparticles. Sol Energy Mater Sol Cells 2014;121:80-4.

[53] Xie F, Choy WCH, Sha EIW, et al. Enhanced charge extraction in organic solar cells through electron accumulation effects induced by metal nanoparticles. Energy Environ Sci 2013;6:3372-9.

[54] Topp K, Borchert H, Johnen F, et al. Impact of the incorporation of Au nanoparticles into polymer/fullerene solar cells. J Phys Chem A 2010;114:3981-9.

[55] Hao Y-W, Wang H-Y, Jiang Y, et al. Hybrid states dynamics of gold nanorods/dye J-aggregate under strong coupling. Angew Chem Int Ed 2011;50:7824-8.
[56] Jiang L, Yin T, Dubrovkin AM, et al. In-plane coherent control of plasmon resonances for plasmonic switching and encoding. Light Sci Appl 2019;8:21.

[57] Su Y-H, Ke Y-F, Cai S-L, Yao Q-Y. Surface plasmon resonance of layer-by-layer gold nanoparticles induced photoelectric current in environmentally-friendly plasmon-sensitized solar cell. Light Sci Appl 2012;1:e14.

[58] Hayashi S, Okamoto T. Plasmonics: visit the past to know the future. J Phys D Appl Phys 2012;45:433001.

[59] Barnes WL, Dereux A, Ebbesen TW. Surface plasmon subwavelength optics. Nature 2003;424:824-30.

[60] Chen F-C, Wu J-L, Lee C-L, Hong Y, Kuo C-H, Huang MH. Plasmonic-enhanced polymer photovoltaic devices incorporating solution-processable metal nanoparticles. Appl Phys Lett 2009;95:013305.

[61] Wang DH, Kim DY, Choi KW, et al. Enhancement of donoracceptor polymer bulk heterojunction solar cell power conversion efficiencies by addition of Au nanoparticles. Angew Chem 2011;50:5519-23.

[62] Kim S-S, Na S-I, Jo J, Kim D-Y, Nah Y-C. Plasmon enhanced performance of organic solar cells using electrodeposited Ag nanoparticles. Appl Phys Lett 2008;93:073307.

[63] Zhang W, Saliba M, Stranks SD, et al. Enhancement of perovskite-based solar cells employing core-shell metal nanoparticles. Nano Lett 2013;13:4505-10.

[64] Lu Z, Pan X, Ma Y, et al. Plasmonic-enhanced perovskite solar cells using alloy popcorn nanoparticles. RSC Adv 2015;5:11175-9.

[65] Mallick SB, Sergeant NP, Agrawal M, Lee J-Y, Peumans P. Coherent light trapping in thin-film photovoltaics. MRS Bull 2011;36:453-60.

[66] Sha WEl, Choy WCH, Liu YG, Chew WC. Near-field multiple scattering effects of plasmonic nanospheres embedded into thin-film organic solar cells. Appl Phys Lett 2011;99:113304.

[67] Kelly KL, Coronado E, Zhao LL, Schatz GC. The optical properties of metal nanoparticles: the influence of size, shape, and dielectric environment. J Phys Chem B 2003;107:668-77.

[68] Bohren CF, Huffman DR. Absorption and scattering of light by small particles. New York: Wiley-Interscience, 1983.

[69] Fan X, Zheng W, Singh DJ. Light scattering and surface plasmons on small spherical particles. Light Sci Appl 2014;3:e179.

[70] Niesen B, Rand BP, Dorpe PV, et al. Near-field interactions between metal nanoparticle surface plasmons and molecular excitons in thin-films. Part I: absorption. J Phys Chem C 2012;116:24206-14.

[71] Weickert J, Dunbar RB, Hesse HC, Wiedemann W, SchmidtMende L. Nanostructured organic and hybrid solar cells. Adv Mater 2011;23:1810-28.

[72] Sha WEI, Choy WCH, Chew WC. Angular response of thin-film organic solar cells with periodic metal back nanostrips. Opt Lett 2011;36:478-80.

[73] Jensen T, Kelly L, Lazarides A, Schatz GC. Electrodynamics of noble metal nanoparticles and nanoparticle clusters. J Cluster Sci 1999;10:295-317.

[74] Nishijima Y, Rosa L, Juodkazis S. Surface plasmon resonances in periodic and random patterns of gold nano-disks for broadband light harvesting. Opt Express 2012;20:11466-77.

[75] Green MA, Pillai S. Harnessing plasmonics for solar cells. Nat Photonics 2012;6:130-2. 
[76] Liu Z, Lee SY, Lee EC. Copper nanoparticle incorporated plasmonic organic bulk-heterojunction solar cells. Appl Phys Lett 2014;105:223306.

[77] Szeremeta J, Nyk M, Chyla A, Strek W, Samoc M. Enhancement of photoconduction in a conjugated polymer through doping with copper nanoparticles. Opt Mater 2011;33:1372-6.

[78] Paci B, Spyropoulos GD, Generosi A, Bailo D, Albertini VR, Stratakis E, Kymakis E. Enhanced structural stability and performance durability of bulk heterojunction photovoltaic devices incorporating metallic nanoparticles. Adv Funct Mater 2011;21:3573-82.

[79] Choy WCH. The emerging multiple metal nanostructures for enhancing the light trapping of thin film organic photovoltaic cells. Chem Commun 2014;50:11984-93.

[80] Back H, Kim G, Kim J, et al. Achieving long-term stable perovskite solar cells via ion neutralization. Energy Environ Sci 2016;9:1258-63.

[81] Akimov YA, Koh WS. Design of plasmonic nanoparticles for efficient subwavelength light trapping in thin-film solar cells. Plasmonics 2011;6:155-61

[82] Duche D, Torchio P, Escoubas L, et al. Improving light absorption in organic solar cells by plasmonic contribution. Sol Energy Mater Sol Cells 2009;93:1377-82.

[83] Brust M, Walker M, Bethell D, Schiffrin DJ, Whyman R. Synthesis of thiol-derivatised gold nanoparticles in a two-phase liquid-liquid system. Chem Commun 1994;7:801-2.

[84] Turkevich J. Colloidal gold. Part II. Gold Bull 1985;18:125-31.

[85] Ackerson CJ, Jadzinsky PD, Kornberg RD. Thiolate ligands for synthesis of water-soluble gold clusters. J Am Chem Soc 2005;127:6550-1.

[86] Louis C, Pluchery O. Gold nanoparticles for physics, chemistry and biology. London: Imperial College Press, 2012.

[87] Frens G. Controlled nucleation for the regulation of the particle size in monodisperse gold suspensions. Nature 1973;241:20-22.

[88] Turkevich J, Stevenson PC, Hillier J. A study of the nucleation and growth processes in the synthesis of colloidal gold. Discuss Faraday Soc 1951;11:55-75.

[89] Jalil SA, Lai B, ElKabbash M, et al. Spectral absorption control of femtosecond laser-treated metals and application in solarthermal devices. Light Sci Appl 2020;9:14.

[90] Yang GW. Laser ablation in liquids: principles and applications in the preparation of nanomaterials. Singapore: Pan Stanford Publications, 2012.

[91] Stratakis E, Barberoglou M, Fotakis C. Generation of Al nanoparticles via ablation of bulk $\mathrm{Al}$ in liquids with short laser pulses. Opt Express 2009;17:12650-9.

[92] Spyropoulos GD, Stylianakis MM, Stratakis E, Kymakis E. Organic bulk heterojunction photovoltaic devices with surfactant-free Au nanoparticles embedded in the active layer. Appl Phys Lett 2012;100:213904.

[93] Barmina EV, Stratakis E, Fotakis C, Shafeev GA. Generation of nanostructures on metals by laser ablation in liquids: new results. Quantum Electron 2010;40:1012.

[94] Day HA, Bartczak D, Fairbairn N, Ardakani M, Porter AE, Kanaras AG. Controlling the three-dimensional morphology of nanocrystals. Cryst Eng Commun 2010;12:4312-6.

[95] Kubiliu-te R, Maximova KA, Lajevardipour A, et al. Ultra-pure, water-dispersed Au nanoparticles produced by femtosecond laser ablation and fragmentation. Int J Nanomed 2013;8:2601.
[96] Chen X, Jia X, Zhang Y, Gu M. Exceeding the limit of plasmonic light trapping in textured screen-printed solar cells using Al nanoparticles and wrinkle-like graphene sheets. Light Sci Appl 2013;2:e92.

[97] Murray WA, Barnes WL. Plasmonic materials. Adv Mater 2007;19:3771-82.

[98] Lee J-S, Cho J, Lee C, et al. Layer-by-layer assembled chargetrap memory devices with adjustable electronic properties. Nat Nanotechnol 2007;2:790-5.

[99] Mallik K, Mandal M, Pradhan N, Pal T. Seed mediated formation of bimetallic nanoparticles by UV irradiation: a photochemical approach for the preparation of "core-shell" type structures. Nano Lett 2001;1:319-22.

[100] Xu M, Feng J, Liu Y-S, Jin Y, Wang H-Y, Sun H-B. Effective and tunable light trapping in bulk heterojunction organic solar cells by employing Au-Ag alloy nanoparticles. Appl Phys Lett 2014;105:153303.

[101] Yao K, Zhong H, Liu Z, et al. Plasmonic metal nanoparticles with core-bishell structure for high-performance organic and perovskite solar cells. ACS Nano 2019;13:5397-409.

[102] Poh C-H, Rosa L, Juodkazis S, Dastoor P. FDTD modeling to enhance the performance of an organic solar cell embedded with gold nanoparticles. Opt Mater Express 2011;1:1326-31.

[103] Xue M, Li L, Villers BJTD, et al. Charge-carrier dynamics in hybrid plasmonic organic solar cells with Ag nanoparticles. Appl Phys Lett 2011;98:253302.

[104] Liu HJ, Goh WP, Leung MY, Li YN, Norsten TB. Effect of nanoparticle stabilizing ligands and ligand-capped gold nanoparticles in polymer solar cells. Sol Energy Mater Sol Cells 2012;96:302-6.

[105] Jankovic V, Yeng Y, You J, et al. Active layer-incorporated, spectrally tuned $\mathrm{Au} / \mathrm{SiO}_{2}$ core/shell nanorod-based light trapping for organic photovoltaics. ACS Nano 2013;7:3815-22.

[106] Chen B, Zhang W, Zhou X, et al. Surface plasmon enhancement of polymer solar cells by penetrating $\mathrm{Au} / \mathrm{SiO}_{2}$ core/shell nanoparticles into all organic layers. Nano Energy 2013;2:906-15.

[107] Xie F-X, Choy WCH, Wang CCD, Sha WEl, Fung DDS. Improving the efficiency of polymer solar cells by incorporating gold nanoparticles into all polymer layers. Appl Phys Lett 2011;99:153304

[108] Wu J-L, Chen F-C, Hsiao Y-S, et al. Surface plasmonic effects of metallic nanoparticles on the performance of polymer bulk heterojunction solar cells. ACS Nano 2011;5:959-67.

[109] Stenzel O, Stendal A, Voigtsberger K, Borczyskowski C. Enhancement of the photovoltaic conversion efficiency of copper phthalocyanine thin film devices by incorporation of metal clusters. Sol Energy Mater Sol Cells 1995;37:337-48.

[110] Yao K, Salvador M, Chueh C-C, et al. A general route to enhance polymer solar cell performance using plasmonic nanoprisms. Adv Energy Mater 2014;4:1400206.

[111] Rand BP, Peumans P, Forrest SR. Long-range absorption enhancement in organic tandem thin-film solar cells containing silver nanoclusters. J Appl Phys 2004;96:7519.

[112] Arinze ES, Qiu B, Nyirjesy G, Thon SM. Plasmonic nanoparticle enhancement of solution-processed solar cells: practical limits and opportunities. ACS Photonics 2016;3:158-73.

[113] Baek S-W, Noh J, Lee C-H, Kim B, Seo M-K, Lee J-Y. Plasmonic forward scattering effect in organic solar cells: a powerful optical engineering method. Sci Rep 2013;3:1726. 
[114] Ham J, Park JY, Dong WJ, Jung GH, Yu HK, Lee JL. Antireflective indium-tin-oxide nanobranches for efficient organic solar cells. Appl Phys Lett 2016;108:073903.

[115] Kim K, Jung B, Kim J, Kim W. Effects of embedding non-absorbing nanoparticles in organic photovoltaics on power conversion efficiency. Sol Energy Mater Sol Cells 2010;94:1835-9.

[116] Ren W, Zhang G, Wu Y, et al. Broadband absorption enhancement achieved by optical layer mediated plasmonic solar cell. Opt Express 2011;19:26536-50.

[117] Lin W-K, Su S-H, Ma C-K, Yokoyama M. Enhancing conversion efficiency of inverted organic solar cells using Ag nanoparticles and long wavelength absorbing tin (II) phthalocyanine. Org Electron 2016;29:94-8.

[118] Segal-Peretz T, Sorias O, Moshonov M, Deckman I, Orenstein M, Frey GL. Plasmonic nanoparticle incorporation into inverted hybrid organic-inorganic solar cells. Org Electron 2015;23:144-50.

[119] Wang DH, Kim JK, Lim G-H, et al. Enhanced light harvesting in bulk heterojunction photovoltaic devices with shapecontrolled Ag nanomaterials: Ag nanoparticles versus Ag nanoplates. RSC Adv 2012;2:7268-72.

[120] Kalfagiannis N, Karagiannidis PG, Pitsalidis C, et al. Plasmonic silver nanoparticles for improved organic solar cells. Sol Energy Mater Sol Cells 2012;104:165-74.

[121] Baek S-W, Park G, Noh J, et al. Au@Ag core-shell nanocubes for efficient plasmonic light scattering effect in low bandgap organic solar cells. ACS Nano 2014;8:3302-12.

[122] Li X, Choy WCH, Huo L, et al. Dual plasmonic nanostructures for high performance inverted organic solar cells. Adv Mater 2012;24:3046-52.

[123] Lu L, Luo Z, Xu T, Yu L. Cooperative plasmonic effect of Ag and Au nanoparticles on enhancing performance of polymer solar cells. Nano Lett 2013;13:59-64.

[124] Bellessa J, Bonnand C, Plenet J, Mugnier J. Strong coupling between surface plasmons and excitons in an organic semiconductor. Phys. Rev. Lett. 2004;93:036404.

[125] Sugawara Y, Kelf T, Baumberg J, Abdelsalam M, Bartlett P. Strong coupling between localized plasmons and organic excitons in metal nanovoids. Phys Rev Lett 2006;97:266808.

[126] Kim G-P, Park B-M, Chang H-J. Effect of Au nano-particles in PEDOT: PSS hole injection layer on the properties of green polymer light-emitting diodes. Electron Mater Lett 2014;10:491-5.

[127] Deibel C, Dyakonov V. Polymer-fullerene bulk heterojunction solar cells. Rep Prog Phys 2010;73:096401.

[128] Wang DH, Park KH, Seo JH, et al. Enhanced power conversion efficiency in PCDTBT/PC70BM bulk heterojunction photovoltaic devices with embedded silver nanoparticle clusters. Adv Energy Mater 2011;1:766-70.

[129] Lee JM, Lim J, Lee N, et al. Synergistic concurrent enhancement of charge generation, dissociation, and transport in organic solar cells with plasmonic metal-carbon nanotube hybrids. Adv Mater 2015;27:1519-25.

[130] Brabec CJ, Gowrisanker S, Halls JJ, Laird D, Jia S, Williams SP. Polymer-fullerene bulk-heterojunction solar cells. Adv Mater 2010;22:3839-56.

[131] Sha WE, Li X, Choy WC. Breaking the space charge limit in organic solar cells by a novel plasmonic-electrical concept. Sci Rep 2014;4:6236.

[132] Sha WE, Zhu HL, Chen L, Chew WC, Choy WC. A general design rule to manipulate photocarrier transport path in solar cells and its realization by the plasmonic-electrical effect. Sci Rep 2015;5:8525.

[133] Ren X, Cheng J, Zhang S, et al. High efficiency organic solar cells achieved by the simultaneous plasmon-optical and plasmon-electrical effects from plasmonic asymmetric modes of gold nanostars. Small 2016;12:5200-7.

[134] Fung DDS, Qiao L, Choy WCH, et al. Optical and electrical properties of efficiency enhanced polymer solar cells with Au nanoparticles in a PEDOT-PSS layer. J Mater Chem 2011;21:16349-56.

[135] Zhang D, Choy WCH, Xie F, et al. Plasmonic electrically functionalized $\mathrm{TiO}_{2}$ for high-performance organic solar cells. Adv Funct Mater 2013;23:4255-61.

[136] Paci B, Generosi A, Albertini VR, Spyropoulos GD, Stratakis E, Kymakis E. Enhancement of photo/thermal stability of organic bulk heterojunction photovoltaic devices via gold nanoparticles doping of the active layer. Nanoscale 2012;4:7452-9.

[137] Kakavelakis G, Stratakis E, Kymakis E. Aluminum nanoparticles for efficient and stable organic photovoltaics. RSC Adv 2013;3:16288-91.

[138] Cho H, Jeong S-H, Park M-H, et al. Overcoming the electroluminescence efficiency limitations of perovskite light-emitting diodes. Science 2015;350:1222-5.

[139] Li Y-F, Chou S-Y, Huang P, et al. Stretchable organometal-halide-perovskite quantum-dot light-emitting diodes. Adv Mater 2019;31:1807516.

[140] Li Y-F, Feng J, Sun H. Perovskite quantum dots for light-emitting devices. Nanoscale 2019;11:19119-39.

[141] Sha WEI, Ren X, Chen L, Choy WCH. The efficiency limit of $\mathrm{CH}_{3} \mathrm{NH}_{3} \mathrm{Pbl}_{3}$ perovskite solar cells. Appl Phys Lett 2015;106:221104.

[142] Ren X, Wang Z, Sha WEI, Choy WCH. Exploring the way to approach the efficiency limit of perovskite solar cells by driftdiffusion model. ACS Photonics 2017;4:934-42.

[143] Saliba M, Zhang W, Burlakov VM, et al. Plasmonic-induced photon recycling in metal halide perovskite solar cells. Adv Funct Mater 2015;25:5038-46.

[144] Hsu H-L, Juang T-Y, Chen C-P, et al. Enhanced efficiency of organic and perovskite photovoltaics from shape-dependent broadband plasmonic effects of silver nanoplates. Sol Energy Mater Sol Cells 2015;140:224-31.

[145] Lee DS, Kim W, Cha BG, et al. Self-position of Au NPs in perovskite solar cells: optical and electrical contribution. ACS Appl Mater Interfaces 2016;8:449-54.

[146] Yue L, Yan B, Attridge M, Wang Z. Light absorption in perovskite solar cell: fundamentals and plasmonic enhancement of infrared band absorption. Solar Energy 2016;124:143-52.

[147] Espinosa N, Serrano-Luján L, Urbina A, Krebs FC. Solution and vapour deposited lead perovskite solar cells: ecotoxicity from a life cycle assessment perspective. Sol Energy Mater Sol Cells 2015;137:303-10.

[148] Cai B, Peng Y, Cheng YB, Gu M. 4-Fold photocurrent enhancement in ultrathin nanoplasmonic perovskite solar cells. Opt Express 2015;23:1700-6.

[149] Green MA, Ho-Baillie A, Snaith HJ. The emergence of perovskite solar cells. Nat Photonics 2014;8:506.

[150] Albrecht S, Saliba M, Baena JPC, et al. Monolithic perovskite/ silicon-heterojunction tandem solar cells processed at low temperature. Energy Environ Sci 2016;9:81-8. 